\title{
On Generalized Progressive Hybrid CENSORING IN PRESENCE OF COMPETING RISKS
}

\author{
Arnab Koley*\& Debasis Kundu*†
}

\begin{abstract}
The progressive Type-II hybrid censoring scheme introduced by Kundu and Joarder (Computational Statistics and Data Analysis, 2509-2528, 2006), has received some attention in the last few years. One major drawback of this censoring scheme is that very few observations (even no observation at all) may be observed at the end of the experiment. To overcome this problem, Cho, Sun and Lee (Statistical Methodology, $23,18-34,2015)$ recently introduced generalized progressive censoring which ensures to get a pre specified number of failures. In this paper we analyze generalized progressive censored data in presence of competing risks. For brevity we have considered only two competing causes of failures, and it is assumed that the lifetime of the competing causes follow one parameter exponential distributions with different scale parameters. We obtain the maximum likelihood estimators of the unknown parameters and also provide their exact distributions. Based on the exact distributions of the maximum likelihood estimators exact confidence intervals can be obtained. Asymptotic and bootstrap confidence intervals are also provided for comparison purposes. We further consider the Bayesian analysis of the unknown parameters under a very flexible Beta-Gamma prior. We provide the Bayes estimates and the associated credible intervals of the unknown parameters based on the above priors. We present extensive simulation results to see the effectiveness of the proposed method and finally one real data set is analyzed for illustrative purpose.
\end{abstract}

Key Words And Phrases: Competing risk; generalized progressive hybrid censoring; beta-gamma distribution; maximum likelihood estimator; bootstrap confidence interval; Bayes credible interval.

AMS Subject Classifications: 62F10, 62F03, $62 \mathrm{H} 12$.

* Department of Mathematics and Statistics, Indian Institute of Technology Kanpur, Pin 208016, India.

${ }^{\dagger}$ Corresponding author, E-mail: kundu@iitk.ac.in, Phone no. 91-512-2597141, Fax no. 91-512-2597500. 


\section{INTRODUCTION}

In many life testing studies, an item or individual may fail due to different causes. In these studies, one observes the time of failure along with it the corresponding cause of failure also. These causes as if compete with each other for the failure of an experimental unit. Hence, in the statistical literature, this is known as the competing risk problem and it has been studied quite extensively by several researchers see for example Kalbfleish and Prentice [23], Lawless [30] and the references cited therein. In a competing risk problem one is naturally interested to find the lifetime distribution of the individual cause, in presence of the other causes. Analysis of the competing risk data is mainly based on one of the two assumptions namely (i) the latent failure time model assumptions of Cox [15] or (ii) the cause specific hazard function assumptions by Prentice et al. [31. Interested readers may refer to the monograph of Crowder [17] for different interesting competing risks problems and for details on different competing risk models.

In life testing experiment most of times the data are censored. The most common censoring schemes used in practice are Type-I and Type-II censoring schemes. Mixture of Type-I and Type-II censoring schemes led to Type-I hybrid censoring scheme which was introduced by Epstein [19] and the Type-II hybrid censoring scheme introduced by Childs et al. [12]. Hybrid censoring schemes have received considerable attention in the last few years, see for example the recent review article by Balakrishnan and Kundu [6] regarding the recent development on this topic.

Note that the censoring schemes outlined above do not allow for removal of units other than the terminal points of the experiments. Cohen [14] was the first one to study more

general censoring scheme known as Progressive (Type-II) censoring scheme. The Progressive censoring scheme can be described as follows. For a fixed sample size $n$ and for fixed effective 
sample size $m$, choose non-negative integers $R_{1}, \ldots, R_{m}$ such that $R_{1}+\ldots+R_{m}=n-m$. The experiment starts with $n$ number of items. At the time of the first failure, $R_{1}$ items are chosen at random from the remaining $n-1$ items and they are removed from the experiment. Similarly, at the time of the second failure, $R_{2}$ items are chosen at random from the remaining $n-R_{1}-2$ items and they are removed from the experiment, and the process continues. Finally, at the time of the $m$-th failure all the remaining items are removed from the system and the experiment stops. Extensive work has been done on different aspects of progressive censoring, see for example the recent book by Balakrishnan and Cramer [3] in this respect.

One major drawback of the progressive Type-II censoring scheme is that the experiment takes a longer time to continue if the units are highly reliable. To overcome that problem, Kundu and Joarder [27] introduced the progressive Type-II hybrid censoring scheme, where a prefixed time point $T$ is introduced along with $n, m$ and $R_{1}, R_{2}, \ldots, R_{m}$. Here the experiment set up is same as that of the progressive type-II censoring scheme except at the termination time point. In this case, the experiment stops at the time point $\min \left\{T, X_{m: m: n}\right\}$, where $X_{m: m: n}$ denotes the failure time of the $m$-th unit. Thus in this set up the experiment never goes beyond the time point $T$. Kundu and Joarder [27] assumed the underlying lifetime distribution to be exponential and developed the inference procedures of the unknown parameters. It was further studied for other lifetime distributions by several authors, see for example Chan [10], Hemmati [21], Cramer [16] and the referenced therein. However in this censoring scheme, the experimenter may observe only few failures or in worst case no failure at all. This will lead to facing the problem of estimating the parameters of the lifetime distributions of the experimental units efficiently.

Recently Cho et al. [13] introduced a new censoring scheme called generalized progressive hybrid censoring. It can be described as follows. The experiment starts with $n$ number of 
items with $k$ and $m$ prefixed integers such that $1 \leq k<m \leq n$. The experimenter chooses predefined time point $T$ and non negative integers $R_{1}, R_{2}, \ldots, R_{m}$ such that $m+R_{1}+$ $R_{2}+\ldots+R_{m}=n$. If $Z_{k: m: n}$ denotes the failure of the $k$-th experimental unit then the experiment stops at the time point $T^{*}=\max \left\{Z_{k: m: n}, \min \left\{T, Z_{m: m: n}\right\}\right\}$. In this paper, we develop the statistical inference of the unknown parameters based on the data coming from a generalized hybrid censoring scheme in presence of competing risk. It is assumed that the lifetime distributions of the competing causes of failures satisfy the latent failure time model assumptions of Cox [15]. It is further assumed that there are only two competing causes, and the lifetime distributions of the competing causes follow one parameter exponential distribution with mean $\theta_{1}$ and $\theta_{2}$, respectively, and they are independently distributed. We obtain the maximum likelihood estimators (MLEs) of the unknown parameters and the exact distributions of the MLEs. Based on the exact distribution, we compute the exact 100(1$\alpha) \%$ confidence intervals of $\theta_{1}$ and $\theta_{2}$. For comparison purposes we compute the bootstrap confidence intervals and the confidence intervals based on the asymptotic distributions of the MLEs. We further consider the Bayesian inference of the unknown parameters based on a very general Beta-Gamma prior. The Bayes estimates and the corresponding credible intervals are also constructed. Extensive simulations are performed to compare the different methods, and one real data set has been analyzed for illustrative purposes.

Rest of the paper is organized as follows. In Section 2, we provide the notations, model description and the MLEs. The exact conditional distributions of the MLEs are provided in Section 3. In Section 4, we provide different confidence intervals of the unknown parameters. The Bayesian inferences are provided in Section 5. In Section 6 we provide simulations results and the analysis of a real data set. Finally we conclude the paper in Section 7 . 


\section{Notations, Model Descriptions And MLEs}

\subsection{Notations}

PDF : probability density function.

$X_{j}: \quad$ random variable associated with the $j$-th cause, $j=1,2$.

$Z: \quad \min \left\{X_{1}, X_{2}\right\}$.

$Z_{i: m: n}: \quad i$-th observed failure of the system, $1 \leq i \leq m$.

$T: \quad$ prefixed time point.

$T^{*}: \quad \max \left\{Z_{k: m: n}, \min \left\{Z_{m: m: n}, T\right\}\right\}$.

$D_{j}: \quad$ number of failures due to cause $j$ for $j=1,2$.

$J$ : number of failures till the time point $T^{*}$.

$R_{i}=$ number of units removed at the time of $i$-th failure, $1 \leq i \leq m$.

$R_{k}^{*}=n-k-\sum_{i=1}^{k-1} R_{i}$.

$R_{J}^{*}=n-J-\sum_{i=1}^{J} R_{i}$.

$\frac{1}{\theta}=\frac{1}{\theta_{1}}+\frac{1}{\theta_{2}}$.

$\gamma_{v}=n-v+1 \sum_{u=1}^{v-1} R_{u}$

$\operatorname{Exp}(\theta): \quad$ exponential distribution with PDF: $\frac{1}{\theta} e^{-\frac{x}{\theta}} ; x>0$.

Gamma $(a, b): \quad$ Gamma distribution with PDF: $\frac{b^{a}}{\Gamma(a)} e^{-b x} x^{a-1} ; x>0$.

$\operatorname{Beta}(a, b): \quad$ Beta distribution with PDF: $\frac{\Gamma(a+b)}{\Gamma(a) \Gamma(b)} x^{a-1}(1-x)^{b-1} ; 0<x<1$.

$f_{G}(x ; a, b, c): \quad$ shifted Gamma distribution with PDF: $\frac{c^{b}}{\Gamma(b)}(x-a)^{b-1} e^{-c(x-a)} ; x>a$. 


\subsection{Model Descriptions, MLEs}

Let $X_{1}$ and $X_{2}$ be the random variables denoting life time distributions of cause 1 and cause 2 , respectively. It is assumed that $X_{i} \sim \operatorname{Exp}\left(\theta_{i}\right)$ for $i=1,2$ and they are independently distributed. At any failure time point one observes $Z=\min \left\{X_{1}, X_{2}\right\}$ and the associated cause of failure. We define a new indicator variable $\delta$ with $\delta_{i}=1$ if the $i$-th failure happens due to Cause- 1 and $\delta_{i}=0$ if the $i$-th failure happens due to Cause-2. The probability density function of $Z$ has the following form,

$$
f\left(z ; \theta_{1}, \theta_{2}\right)= \begin{cases}\left(\frac{1}{\theta_{1}}+\frac{1}{\theta_{2}}\right) e^{-z\left(\frac{1}{\theta_{1}}+\frac{1}{\theta_{2}}\right)} ; & \text { if } z>0 \\ 0 ; & \text { otherwise }\end{cases}
$$

Under the generalized progressive hybrid censoring scheme, the possible values of $T^{*}$ are

$$
T^{*}=\left\{\begin{array}{lll}
T & \text { if } & Z_{k: m: n}<T<Z_{m: m: n} \\
Z_{k: m: n} & \text { if } \quad T<Z_{k: m: n}<Z_{m: m: n} \\
Z_{m: m: n} & \text { if } & Z_{k: m: n}<Z_{m: m: n}<T
\end{array}\right.
$$

It is to be noted that, the likelihood contribution at the point $(z, \delta=1)$ is given by,

$$
L\left(\theta_{1}, \theta_{2} \mid(z, \delta=1)\right)=\frac{1}{\theta_{1}} e^{-\frac{1}{\theta_{1}} z} e^{-\frac{1}{\theta_{2}} z}=\frac{1}{\theta_{1}} e^{-\left(\frac{1}{\theta_{1}}+\frac{1}{\theta_{2}}\right) z} .
$$

Similarly, the likelihood contribution at the point $(z, \delta=0)$ is given by,

$$
L\left(\theta_{1}, \theta_{2} \mid(z, \delta=0)\right)=\frac{1}{\theta_{2}} e^{-\frac{1}{\theta_{2}} z} e^{-\frac{1}{\theta_{1}} z}=\frac{1}{\theta_{2}} e^{-\left(\frac{1}{\theta_{1}}+\frac{1}{\theta_{2}}\right) z}
$$

Hence, based on the observations, the likelihood function can be written as,

$$
L\left(\theta_{1}, \theta_{2} \mid \text { Data }\right)=c\left(\frac{1}{\theta_{1}}\right)^{D_{1}}\left(\frac{1}{\theta_{2}}\right)^{D_{2}} e^{-\frac{W}{\theta}},
$$

where,

$$
\begin{gathered}
c= \begin{cases}\prod_{v=1}^{J} \gamma_{v}, & \text { if } T^{*}=T, \\
\prod_{v=1}^{k} \gamma_{v}, & \text { if } T^{*}=Z_{k: m: n}, \\
\prod_{v=1}^{m} \gamma_{v}, & \text { if } T^{*}=Z_{m: m: n},\end{cases} \\
\gamma_{v}=n-v+1 \sum_{u=1}^{v-1} R_{u}, \quad v \in\{1,2, \ldots, m\},
\end{gathered}
$$




$$
W= \begin{cases}\sum_{i=1}^{J} z_{i}\left(1+R_{i}\right)+T R_{J}^{*}, & \text { if } T^{*}=T, \\ \sum_{i=1}^{k-1} z_{i}\left(1+R_{i}\right)+z_{k}\left(1+R_{k}^{*}\right), & \text { if } T^{*}=Z_{k: m: n}, \\ \sum_{i=1}^{m} z_{i}\left(1+R_{i}\right), & \text { if } T^{*}=Z_{m: m: n}\end{cases}
$$

Note that, $\gamma_{v}$ denotes the number of units remaining at the time of $v$-th failure. Clearly, $J=k, k+1, \ldots, m-1$ when $T^{*}=T$. Thus the log likelihood function (ignoring the constant c) is given by,

$$
l\left(\theta_{1}, \theta_{2}\right)=-D_{1} \log \theta_{1}-D_{2} \log \theta_{2}-\frac{W}{\theta}
$$

Note that the MLE of $\theta_{1}\left(\theta_{2}\right)$ exists if $D_{1}>0\left(D_{2}>0\right)$. If $D_{1}=0\left(D_{2}=0\right)$ then the likelihood function is unbounded above as a function of $\theta_{1}\left(\theta_{2}\right)$ and thus the MLE of $\theta_{1}$ $\left(\theta_{2}\right)$ does not exist. Taking partial derivatives of equation (2) with respect to $\theta_{1}$ and $\theta_{2}$ and equating them to 0 we get MLEs of $\theta_{1}$ (given, $D_{1}>0$ ) and $\theta_{2}$ (given, $D_{2}>0$ ) as

$$
\widehat{\theta}_{1}=\frac{W}{D_{1}} \text { and } \widehat{\theta}_{2}=\frac{W}{D_{2}}
$$

\section{Main Result: Exact Conditional distributions of THE MLES}

In this section we provide the exact marginal distributions of the MLEs of the parameters $\theta_{1}$ and $\theta_{2}$ conditioning on $D_{1}>0$ and $D_{2}>0$, respectively. 
Theorem 1. The conditional PDF of $\widehat{\theta}_{1}$ conditioning on $D_{1}>0$, is given by

$$
\begin{aligned}
& f_{\widehat{\theta}_{1} \mid D_{1}>0}(x)=\frac{1}{P\left(D_{1}>0\right)}\left[\sum _ { j = k } ^ { m - 1 } \sum _ { i = 1 } ^ { j } \left\{\prod_{v=1}^{j} \gamma_{v}\left(\frac{1}{\theta_{1}}\right)^{i}\left(\frac{1}{\theta_{2}}\right)^{j-i}\left(\frac{1}{\theta}\right)^{-j}\right.\right. \\
& \sum_{v=0}^{j} \frac{(-1)^{v} e^{-\frac{T}{\theta} \gamma_{j-v+1}}}{\left\{\prod_{h=1}^{v}\left(\gamma_{j+1-v}-\gamma_{j+1-v+h}\right)\right\}\left\{\prod_{h=1}^{j-v}\left(\gamma_{h}-\gamma_{j-v+1}\right)\right\}} \\
& \left.f_{G}\left(x ; \frac{T}{i} \gamma_{j-v+1}, j, \frac{i}{\theta}\right)\right\} \\
& +\sum_{i=1}^{k}\left\{\prod_{v=1}^{k} \gamma_{v}\left(\frac{1}{\theta_{1}}\right)^{i}\left(\frac{1}{\theta_{2}}\right)^{k-i}\left(\frac{1}{\theta}\right)^{-k}\right. \\
& \sum_{v=0}^{k-1} \frac{(-1)^{v} e^{-\frac{T}{\theta} \gamma_{k-v}}}{\left\{\prod_{j=1}^{v}\left(\gamma_{k-v}-\gamma_{k-v+j}\right)\right\}\left\{\prod_{j=1}^{k-1-v}\left(\gamma_{j}-\gamma_{k-v}\right)\right\} \gamma_{k-v}} \\
& \left.f_{G}\left(x ; \frac{T}{i} \gamma_{k-v}, k, \frac{i}{\theta}\right)\right\} \\
& +\sum_{i=1}^{m}\left\{\prod_{v=1}^{m} \gamma_{v}\left(\frac{1}{\theta_{1}}\right)^{i}\left(\frac{1}{\theta_{2}}\right)^{m-i}\left(\frac{1}{\theta}\right)^{-m}\right. \\
& \sum_{v=0}^{m} \frac{(-1)^{v} e^{-\frac{T}{\theta}\left(\gamma_{m-v+1}-\gamma_{m+1}\right)}}{\left\{\prod_{j=1}^{v}\left(\gamma_{m-v+1}-\gamma_{m-v+j+1}\right)\right\}\left\{\prod_{j=1}^{m-v}\left(\gamma_{j}-\gamma_{m-v+1}\right)\right\}} \\
& \left.\left.f_{G}\left(x ; \frac{T}{i}\left(\gamma_{m-v+1}-\gamma_{m+1}\right), m, \frac{i}{\theta}\right)\right\}\right] \text {, }
\end{aligned}
$$

where,

$$
P\left(D_{1}>0\right)=1-P\left(D_{1}=0\right)
$$

and

$$
\begin{aligned}
P\left(D_{1}=0\right)= & \sum_{j=k}^{m} \prod_{v=1}^{j+1} \sum_{u=0}^{j} \frac{\gamma_{v}(-1)^{u} e^{-\frac{T}{\theta} \gamma_{j-u+1}}}{\left\{\prod_{v=1}^{u}\left(\gamma_{j+1-u}-\gamma_{j+1-u+v}\right)\right\}\left\{\prod_{v=1}^{j-u}\left(\gamma_{v}-\gamma_{j-u+1}\right)\right\}\left\{\gamma_{j-u+1}-u\right\}}\left(\frac{\theta_{1}}{\theta_{1}+\theta_{2}}\right)^{j} \\
& +\prod_{v=1}^{k} \sum_{v=0}^{k-1} \frac{\gamma_{v}(-1)^{v} e^{-\frac{T}{\theta} \gamma_{k-v}}}{\left\{\prod_{j=1}^{v}\left(\gamma_{k-v}-\gamma_{k-v+j}\right)\right\}\left\{\prod_{j=1}^{k-1-v}\left(\gamma_{j}-\gamma_{k-v}\right)\right\}\left\{\gamma_{k-v}\right\}}\left(\frac{\theta_{1}}{\theta_{1}+\theta_{2}}\right)^{k} \\
& +\prod_{v=1}^{m} \sum_{v=0}^{m} \frac{\gamma_{v}(-1)^{v} e^{-\frac{T}{\theta}\left(\gamma_{m-v+1}-\gamma_{m+1}\right)}}{\left\{\prod_{j=1}^{v}\left(\gamma_{m-v+1}-\gamma_{m-v+j+1}\right)\right\}\left\{\prod_{j=1}^{m-v}\left(\gamma_{j}-\gamma_{m-v+1}\right)\right\}}\left(\frac{\theta_{1}}{\theta_{1}+\theta_{2}}\right)^{m}
\end{aligned}
$$




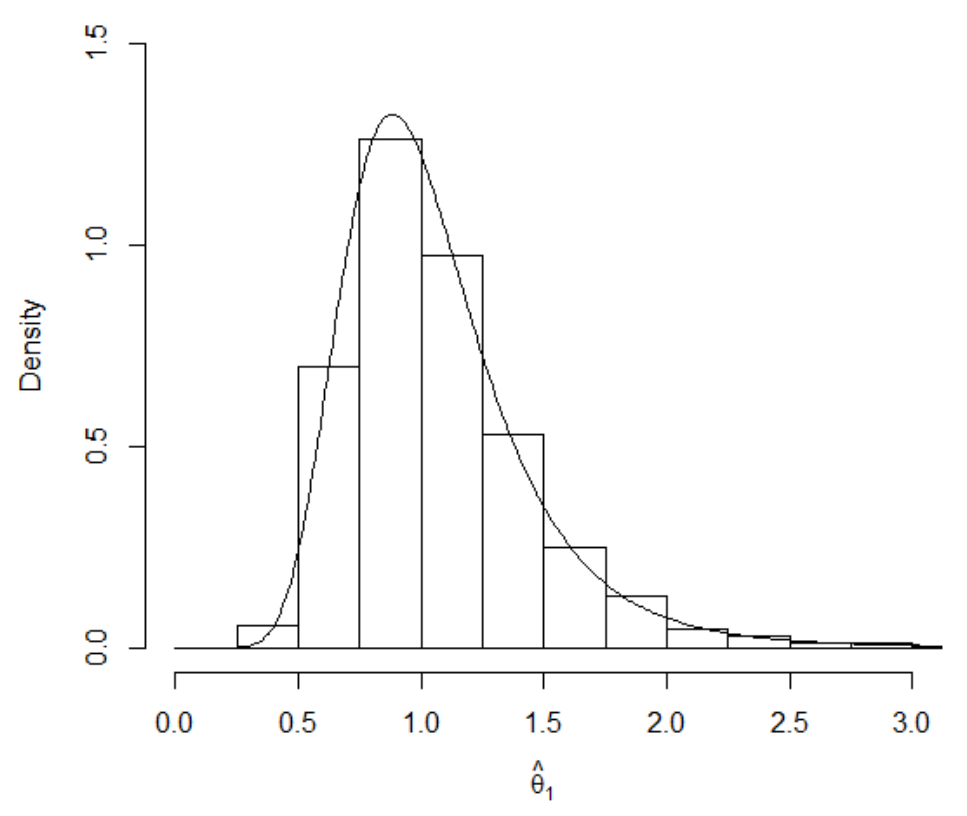

Figure 1: Histogram of $\widehat{\theta}_{1}$ along its PDF

Proof. See in the Appendix.

Similarly, the conditional distribution of $\widehat{\theta}_{2}$ given $D_{2}>0$ is obtained by replacing $D_{1}$ and $\theta_{1}$ by $D_{2}$ and $\theta_{2}$ respectively.

Comment: The conditional PDFs of $\widehat{\theta}_{1}$ and $\widehat{\theta}_{2}$ are quite complicated. To get some idea about the shape of the PDFs, we plot the conditional PDF of $\widehat{\theta}_{1}$ and $\widehat{\theta}_{2}$ in Figure 1 and Figure 2, respectively for $n=20, k=5, m=18, T=1.2, \theta_{1}=1.0, \theta_{2}=1.3$, and for the censoring Scheme-III (see Section 6 for details). We have also plotted the histograms of $\widehat{\theta}_{1}$ and $\widehat{\theta}_{2}$ based on 5000 replications on the same graph, and they match very well. It verifies the correctness of the expressions of the conditional distributions of $\widehat{\theta}_{1}$ and $\widehat{\theta}_{2}$. 


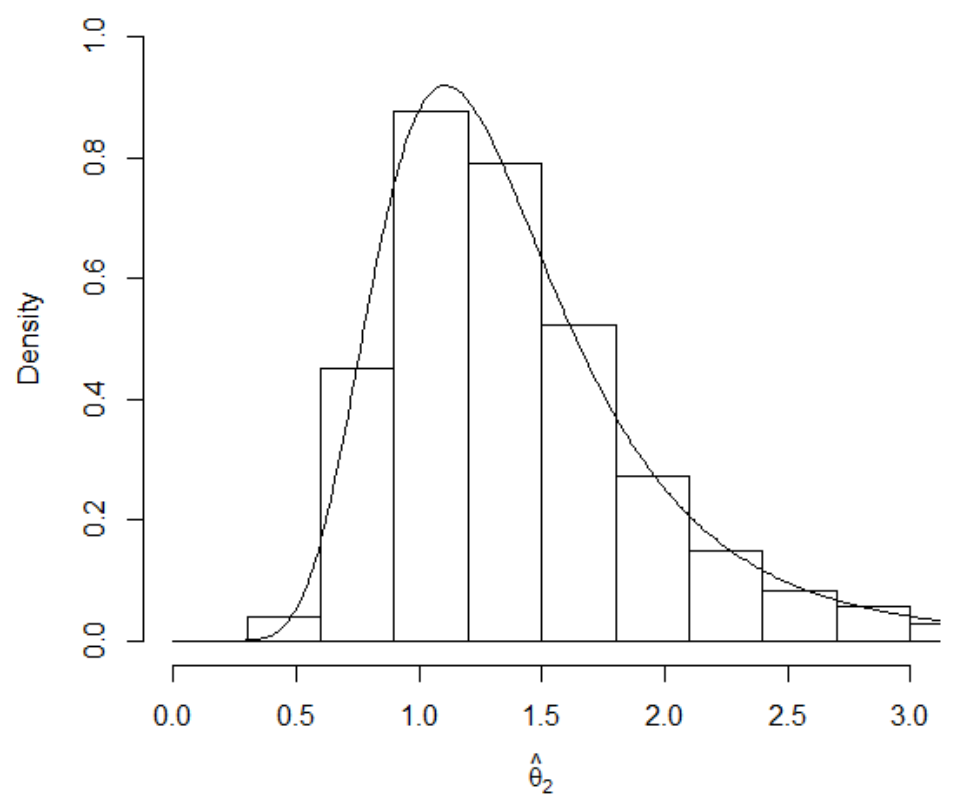

Figure 2: Histogram of $\widehat{\theta}_{2}$ along its PDF

\section{Confidence interval}

From the exact conditional distribution of $\widehat{\theta}_{1}$, the exact CI can be constructed under the assumption that $P_{\theta_{1}}\left(\widehat{\theta}_{1} \leq x\right)$ is a monotonic decreasing function of $\theta_{1}$ for fixed $x$. Several authors including Balakrishnan et al. [7], Chen and Bhattayacharya [11], Childs et al. [12], Kundu and Basu [25] used this technique to construct CI for the parameters. Although we

cannot prove the monotonicity property of $P_{\theta_{1}}\left(\widehat{\theta}_{1} \leq x\right)$ analytically, a graphical plot supports this property, see Figure 3 and Figure 4 .

The confidence interval of $\theta_{1}$ can be obtained as follows, see Kundu and Basu [25] for details. Suppose, $\widehat{\theta}_{1, \text { obs }}$ is an estimated value of $\theta_{1}$. Then for $\alpha \in(0,1)$, a $100(1-\alpha) \%$ exact CI is given by $\left(\theta_{1 l}, \theta_{1 u}\right)$ where, $\theta_{1 l}$ and $\theta_{1 u}$ are obtained from the following two equations,

$$
P_{\theta_{1 l}}\left(\widehat{\theta}_{1} \leq \widehat{\theta}_{1, o b s}\right)=1-\frac{\alpha}{2} \quad \text { and } \quad P_{\theta_{1 u}}\left(\widehat{\theta}_{1} \leq \widehat{\theta}_{1, o b s}\right)=\frac{\alpha}{2}
$$




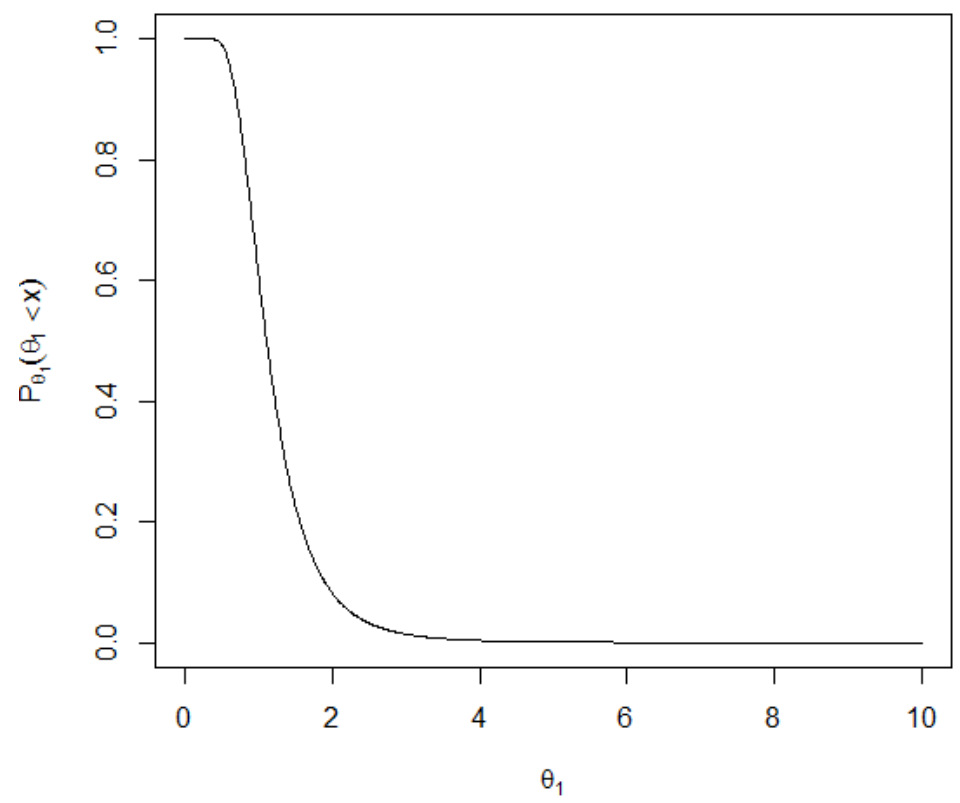

Figure 3: The plot of $P_{\theta_{1}}\left(\widehat{\theta}_{1} \leq x\right)$ for $n=20, k=3, m=14, T=1.2, x=1.109, \theta_{2}=1.472$ under Scheme-I

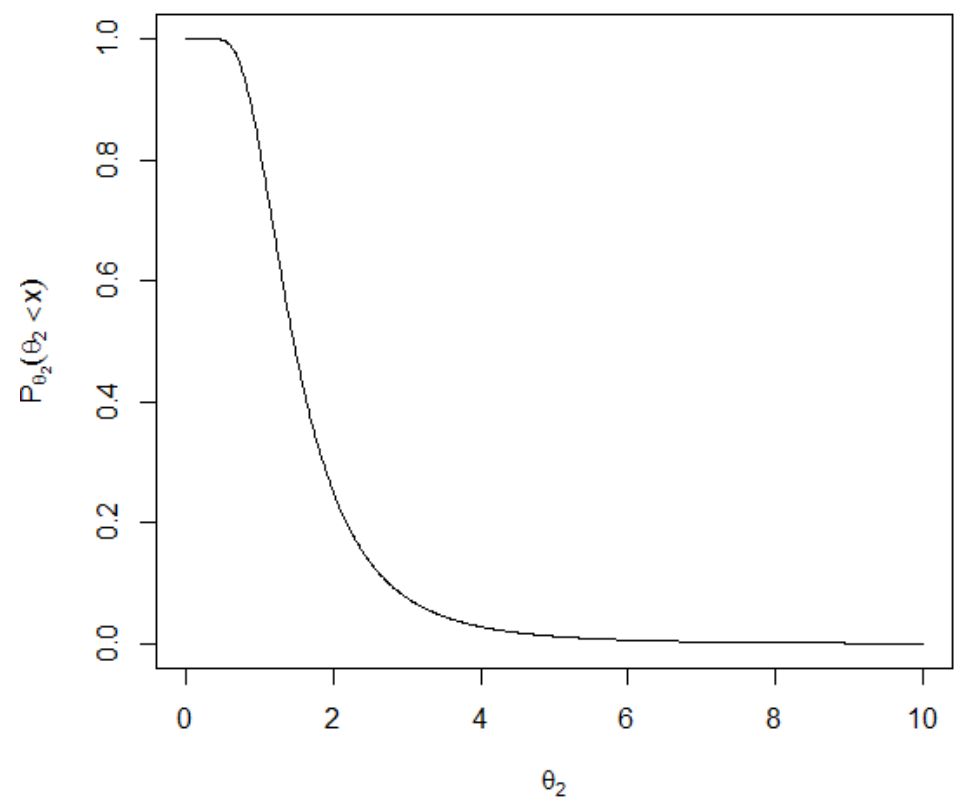

Figure 4: The plot of $P_{\theta_{2}}\left(\widehat{\theta}_{2} \leq x\right)$ for $n=20, k=3, m=14, T=1.2, x=1.472, \theta_{1}=1.109$ under Scheme-I 
Clearly due to complicated nature of PDF of $\widehat{\theta}_{1}$, the above two equations turn out to be non linear equations. One needs to solve them numerically, say for example by Newton Rapshon method or Bisection method. Similarly we can obtain the confidence interval for $\theta_{2}$ also.

It should be mentioned that the exact confidence intervals of $\theta_{1}$ and $\theta_{2}$ may not always exist, see for example Balakrishnan et al. [4] for all $0<\alpha<1$. In fact it is clear that if $P_{\theta_{1}}\left(\widehat{\theta}_{1} \leq \widehat{\theta}_{1, o b s}\right)$ varies from 1 to 0 , as $\theta_{1}$ varies from 0 to infinity, for all values of $\widehat{\theta}_{1, \text { obs }}$, then the exact confidence interval of $\theta_{1}$ exists for all values of $0<\alpha<1$. Similarly, for $\theta_{2}$ also. It is not difficult to show that $\lim _{\theta_{1} \rightarrow 0} P_{\theta_{1}}\left(\widehat{\theta}_{1} \leq \widehat{\theta}_{1, \text { obs }}\right)=1$ and $\lim _{\theta_{2} \rightarrow 0} P_{\theta_{2}}\left(\widehat{\theta}_{2} \leq \widehat{\theta}_{2, o b s}\right)=1$. But we could not establish the following limits $\lim _{\theta_{1} \rightarrow \infty} P_{\theta_{1}}\left(\widehat{\theta}_{1} \leq \widehat{\theta}_{1, o b s}\right)$ and $\lim _{\theta_{2} \rightarrow \infty} P_{\theta_{2}}\left(\widehat{\theta}_{2} \leq \widehat{\theta}_{2, o b s}\right)$. In fact it has been observed in our simulation experiments that these limits may not be 0 always, and in those cases the exact finite length confidence intervals do not exist for all $0<\alpha<1$. We have demonstrated that in Section 6 .

Due to complicated functional form of the exact conditional distribution function, it is quite difficult to obtain exact confidence intervals of the parameters in practice. Hence, we propose to use bootstrap method for construction of CIs of the unknown parameters. The steps are similar as given in Kundu and Gupta [26], hence they are omitted.

\section{Bayesian Analysis}

In this section we consider the Bayesian inference of the unknown parameters. We obtain the Bayes estimates and the highest posterior density (HPD) credible intervals of the unknown parameters. Suppose, $\lambda_{1}=\frac{1}{\theta_{1}}$ and $\lambda_{2}=\frac{1}{\theta_{2}}$, we take a joint prior distribution on $\left(\lambda_{1}, \lambda_{2}\right)$ as the Beta-gamma distribution, see for example Pena and Gupta [29], with parameters $b_{0}>0, a_{0}>0, a_{1}>0, a_{2}>0$. From now on it will be denoted by $\mathrm{BG}\left(b_{0}, a_{0}, a_{1}, a_{2}\right)$ and it 
has the joint PDF for $\lambda_{1}, \lambda_{2}>0$ as follows;

$$
\pi\left(\lambda_{1}, \lambda_{2} \mid b_{0}, a_{0}, a_{1}, a_{2}\right)=\frac{\Gamma\left(a_{1}+a_{2}\right)}{\Gamma\left(a_{0}\right)}\left(b_{0}\left(\lambda_{1}+\lambda_{2}\right)\right)^{a_{0}-a_{1}-a_{2}} \frac{b_{0}^{a_{1}}}{\Gamma\left(a_{1}\right)} \lambda_{1}^{a_{1}-1} e^{-b_{0} \lambda_{1}} \frac{b_{0}^{a_{2}}}{\Gamma\left(a_{2}\right)} \lambda_{2}^{a_{2}-1} e^{-b_{0} \lambda_{2}} .
$$

The joint posterior distribution of $\left(\lambda_{1}, \lambda_{2}\right)$ is given by,

$$
\pi\left(\lambda_{1}, \lambda_{2} \mid \text { data }\right) \propto e^{-\left(W+b_{0}\right)\left(\lambda_{1}+\lambda_{2}\right)} \lambda_{1}^{a_{1}+D_{1}-1} \lambda_{2}^{a_{2}+D_{2}-1}\left(\lambda_{1}+\lambda_{2}\right)^{a_{0}-a_{1}-a_{2}} ; \quad \lambda_{1}>0, \lambda_{2}>0,
$$

and it is $\mathrm{BG}\left(W+b_{0}, a_{0}+D_{1}+D_{2}, a_{1}+D_{1}, a_{2}+D_{2}\right)$. The Bayes estimates of $\theta_{1}$ and $\theta_{2}$ under square error loss function are, respectively,

$$
\widehat{\theta}_{1 B}=E\left(\frac{1}{\lambda_{1}} \mid \text { data }\right)=\frac{\left(a_{1}+a_{2}+D_{1}+D_{2}-1\right)\left(w+b_{0}\right)}{\left(a_{0}+D_{1}+D_{2}-1\right)\left(a_{1}+D_{1}-1\right)}
$$

and

$$
\widehat{\theta}_{2 B}=E\left(\frac{1}{\lambda_{2}} \mid \text { data }\right)=\frac{\left(a_{1}+a_{2}+D_{1}+D_{2}-1\right)\left(w+b_{0}\right)}{\left(a_{0}+D_{1}+D_{2}-1\right)\left(a_{2}+D_{2}-1\right)} .
$$

The corresponding posterior variances are, respectively,

$$
V\left(\theta_{1} \mid \text { Data }\right)=A_{1} B_{1} \text { and } \quad V\left(\theta_{2} \mid \text { Data }\right)=A_{2} B_{2}
$$

where for $k=1,2$,

$$
A_{k}=\frac{\left(w+b_{0}\right)^{2}\left(a_{1}+a_{2}+D_{1}+D_{2}-1\right)}{\left(a_{0}+D_{1}+D_{2}-1\right)\left(a_{k}+D_{k}-1\right)}
$$

and

$$
B_{k}=\frac{\left(a_{1}+a_{2}+D_{1}+D_{2}-2\right)}{\left(a_{0}+D_{1}+D_{2}-2\right)\left(a_{k}+D_{k}-2\right)}-\frac{\left(a_{1}+a_{2}+D_{1}+D_{2}-1\right)}{\left(a_{0}+D_{1}+D_{2}-1\right)\left(a+k+D_{k}-1\right)} .
$$

\subsection{CREDIBLE SET}

Let us recall that for $\alpha \in(0,1)$, a set $C_{\alpha}$ is said to be a $100(1-\alpha) \%$ credible set for $\left(\lambda_{1}, \lambda_{2}\right)$ if $P\left[\left(\lambda_{1}, \lambda_{2}\right) \in C_{\alpha}\right]=1-\alpha$, where $\left(\lambda_{1}, \lambda_{2}\right) \sim \pi\left(\lambda_{1}, \lambda_{2} \mid\right.$ data $)$. To construct a $100(1-\alpha) \%$ credible set we use the following theorem. The proof is quite straightforward, hence it is not provided here. 
Theorem 2. If $\left(\lambda_{1}, \lambda_{2}\right) \sim B G\left(b_{0}+W, a_{0}+J, a_{1}+D_{1}, a_{2}+D_{2}\right)$ then,

$$
U=\lambda_{1}+\lambda_{2} \sim \operatorname{Gamma}\left(a_{0}+D_{1}+D_{2}, b_{0}+W\right), \quad V=\frac{\lambda_{1}}{\lambda_{1}+\lambda_{2}} \sim \operatorname{Beta}\left(a_{1}+D_{1}, a_{2}+D_{2}\right)
$$

and they are independently distributed.

Using the above theorem we construct a $100(1-\alpha) \%$ credible set of $\left(\lambda_{1}, \lambda_{2}\right)$ as follows. Let $0<\alpha_{1}, \alpha_{2}<1$ such that $\left(1-\alpha_{1}\right)\left(1-\alpha_{2}\right)=1-\alpha$. Then $C_{\alpha}$ can be constructed as,

$$
C_{\lambda_{1}, \lambda_{2}, \alpha}=\left\{\left(\lambda_{1}, \lambda_{2}\right): A<\lambda_{1}+\lambda_{2}<B, C<\frac{\lambda_{1}}{\lambda_{1}+\lambda_{2}}<D\right\}
$$

with $P\left(A<\lambda_{1}+\lambda_{2}<B\right)=1-\alpha_{1}$ and $P\left(C<\frac{\lambda_{1}}{\lambda_{1}+\lambda_{2}}<D\right)=1-\alpha_{2}$.

Note that, $C_{\lambda_{1}, \lambda_{2}, \alpha}$ is a trapezoid enclosed by the following four straight lines:

(i) $\lambda_{1}+\lambda_{2}=A, \quad(i i) \lambda_{1}+\lambda_{2}=B, \quad(i i i) \lambda_{1}(1-D)=\lambda_{2} D, \quad(i v) \lambda_{1}(1-C)=\lambda_{2} C$.

Equivalently, we can get a $100(1-\alpha) \%$ credible set of $\left(\theta_{1}, \theta_{2}\right)$ as follows

$$
C_{\theta_{1}, \theta_{2}, \alpha}=\left\{\left(\theta_{1}, \theta_{2}\right): A<\frac{\theta_{1}+\theta_{2}}{\theta_{1} \theta_{2}}<B, C<\frac{\theta_{2}}{\theta_{1}+\theta_{2}}<D\right\}
$$

Hence, $C_{\theta_{1}, \theta_{2}, \alpha}$ is an area enclosed by the following four lines (two of them are straight lines and two of them are curved lines).

(i) $\theta_{1}+\theta_{2}=A \theta_{1} \theta_{2}, \quad(i i) \theta_{1}+\theta_{2}=B \theta_{1} \theta_{2}, \quad(i i i) \theta_{1} D=\theta_{2}(1-D), \quad(i v) \theta_{1} C=\theta_{2}(1-C)$.

Although, we can obtain a $100(1-\alpha) \%$ credible set of $\left(\lambda_{1}, \lambda_{2}\right)$ or of $\left(\theta_{1}, \theta_{2}\right)$ by the above method, it is not possible to obtain the HPD credible intervals for any function of $\lambda_{1}, \lambda_{2}$ or of $\theta_{1}, \theta_{2}$. We propose to use the Gibbs sampling technique to construct the $100(1-\alpha) \%$ HPD credible interval of any function of $\lambda_{1}$ and $\lambda_{2}$, say, $g\left(\lambda_{1}, \lambda_{2}\right)$. Shrijita et al. [9] gave similar algorithm to find HPD credible interval of $g\left(\lambda_{1}, \lambda_{2}\right)$ and hence are omitted here. 


\section{Simulation and Data Analysis}

\subsection{Simulation}

In this section we present some simulation results mainly to see how the different methods proposed in this paper behave in practice. We have kept $\theta_{1}=1, \theta_{2}=1.3, T=1.2$ fixed. We have considered different sample sizes $(n)$, different effective sample sizes $(m)$, different $k$ values and three different progressive censoring schemes namely; Scheme-I $\left(R_{1}=n-m\right.$, $\left.R_{2}=\ldots=R_{m}=0\right)$, Scheme-II $\left(R_{m / 2}=0, R_{1}=\ldots=R_{m / 2-1}=R_{m / 2+1}=\ldots=R_{m}=\right.$ $0)$ and Scheme-III $\left(R_{1}=\ldots=R_{m-1}=0, R_{m}=n-m\right)$. We have considered the following different cases, see Table 1. In each case we compute the MLEs of the unknown parameters,

Table 1: Different cases considered for simulation experiments.

\begin{tabular}{|c|c|c|}
\hline Scheme-I & Scheme-II & Scheme-III \\
\hline $\mathrm{n}=20, \mathrm{k}=3, \mathrm{~m}=14$ & $\mathrm{n}=20, \mathrm{k}=3, \mathrm{~m}=14$ & $\mathrm{n}=20, \mathrm{k}=3, \mathrm{~m}=14$ \\
$\mathrm{n}=20, \mathrm{k}=5, \mathrm{~m}=14$ & $\mathrm{n}=20, \mathrm{k}=5, \mathrm{~m}=14$ & $\mathrm{n}=20, \mathrm{k}=5, \mathrm{~m}=14$ \\
$\mathrm{n}=20, \mathrm{k}=3, \mathrm{~m}=18$ & $\mathrm{n}=20, \mathrm{k}=3, \mathrm{~m}=18$ & $\mathrm{n}=20, \mathrm{k}=3, \mathrm{~m}=18$ \\
$\mathrm{n}=20, \mathrm{k}=5, \mathrm{~m}=18$ & $\mathrm{n}=20, \mathrm{k}=5, \mathrm{~m}=18$ & $\mathrm{n}=20, \mathrm{k}=5, \mathrm{~m}=18$ \\
$\mathrm{n}=30, \mathrm{k}=6, \mathrm{~m}=22$ & $\mathrm{n}=30, \mathrm{k}=6, \mathrm{~m}=22$ & $\mathrm{n}=30, \mathrm{k}=6, \mathrm{~m}=22$ \\
$\mathrm{n}=30, \mathrm{k}=8, \mathrm{~m}=22$ & $\mathrm{n}=30, \mathrm{k}=8, \mathrm{~m}=22$ & $\mathrm{n}=30, \mathrm{k}=8, \mathrm{~m}=22$ \\
$\mathrm{n}=30, \mathrm{k}=6, \mathrm{~m}=26$ & $\mathrm{n}=30, \mathrm{k}=6, \mathrm{~m}=26$ & $\mathrm{n}=30, \mathrm{k}=6, \mathrm{~m}=26$ \\
$\mathrm{n}=30, \mathrm{k}=8, \mathrm{~m}=26$ & $\mathrm{n}=30, \mathrm{k}=8, \mathrm{~m}=26$ & $\mathrm{n}=30, \mathrm{k}=8, \mathrm{~m}=26$ \\
\hline
\end{tabular}

and construct the $95 \%$ exact confidence intervals and the bootstrap confidence intervals for both the parameters. We replicate the process 5000 times, and report the average biases, mean squared errors (MSE), the average length of the confidence intervals and the associated coverage percentages within brackets.

One important point we would like to mention that a finite length of $95 \%$ exact confidence interval of each of the parameters does not exist for each replication. This happens when the number of failures due to a particular cause is very low (1 or 2 mostly). We have reported the number of times the exact confidence intervals do not exist (NECI) also. The results are 
presented in Table 2 and Table 5. We give few graphical evidences of such non existence of solutions [see Figure 5 and Figure 6] .

To perform the Bayesian analysis we consider two different priors; Prior-I (informative) and Prior-II (matching prior). For Prior-I, the hyper parameters are $a_{0}=46 / 13, b_{0}=1, a_{1}=$ 2.3, $a_{2}=2$, and they have been chosen in such a manner that $E\left(\lambda_{1}\right)=1$ and $E\left(\lambda_{2}\right)=1 / 1.3$, the true values of the parameters considered for simulation experiments. For Prior-II, the hyper parameters are $a_{0}=2, b_{0}=0, a_{1}=1, a_{2}=1$, and they have been chosen in such a manner that the Bayes estimates match with the corresponding MLEs. In this case also similarly as before, in each case we compute the Bayes estimates and the symmetric and HPD credible intervals. We replicate the process 5000 times, and report the average biases, MSEs, the average lengths of the credible intervals and the associated coverage percentages within brackets. The results are presented in Table 3, Table 4, Table 6] and Table 7 .

Some of the points are quite clear from these simulation results. It is observed that the performance of the MLEs are quite satisfactory. As the sample size increase the biases and the MSEs decrease as expected. Comparing the performances of the different confidence intervals it is observed that the performances of the exact confidence intervals and the bootstrap confidence intervals are quite good. In both these cases coverage percentage are maintained. Since the average lengths of the bootstrap confidence intervals are slightly smaller than the corresponding average lengths of the exact confidence intervals we propose to use the bootstrap confidence intervals for all practical purposes, and they are very easy to implement also.

Clearly, from Figure 5 and Figure 6 we see that upper confidence limits of the parameters $\theta_{1}$ and $\theta_{2}$ do not exist for large estimates of the corresponding parameters. In other words, it can be said that finite length of confidence interval does not exist for $\theta_{1}$ when $D_{1}$ is very small. Similar case holds for $\theta_{2}$ also. 


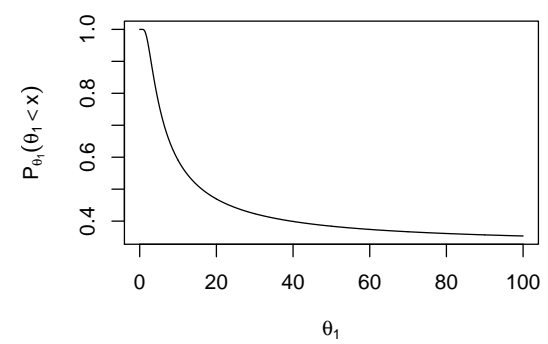

Figure 5: The plot of $P_{\theta_{1}}\left(\widehat{\theta}_{1} \leq x\right)$ for $n=20, k=3, m=14, T=1.2, x=7.549, \theta_{2}=0.755$ under Scheme-I

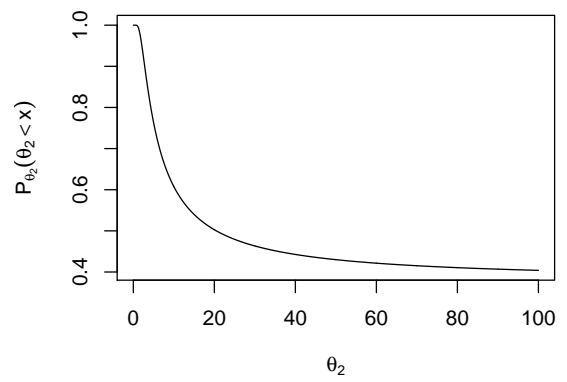

Figure 6: The plot of $P_{\theta_{2}}\left(\widehat{\theta}_{2} \leq x\right)$ for $n=20, k=3, m=14, T=1.2, x=6.864, \theta_{1}=0.624$ under Scheme-I

The performance of the Bayes estimates also are quite satisfactory. The average biases and MSEs of the Bayes estimates under Prior-II match with the corresponding values associated with the MLEs, as it should be. In case of Prior-I the average biases and the associated MSEs are slightly smaller than Prior-II. Regarding the credible intervals it is clear that both the symmetric and HPD credible intervals are performing quite well. Since the coverage percentages for the symmetric credible intervals are closer to the nominal value, it might be preferable compared to the HPD credible intervals. Hence, if we do not have any prior information, then use the Bayes estimates with the matching prior, and if we have any prior information, then use the informative prior. 
Table 2: Results of frequentist analysis for $\theta_{1}$

\begin{tabular}{|c|c|c|c|c|c|c|c|c|}
\hline $\mathrm{n}$ & $\mathrm{m}$ & $\mathrm{k}$ & Scheme & Bias & MSE & $\begin{array}{c}\text { Exact } \\
\text { CI }\end{array}$ & NECI & $\begin{array}{c}\text { Bootstrap } \\
\text { CI }\end{array}$ \\
\hline \multirow[t]{12}{*}{20} & \multirow[t]{6}{*}{14} & \multirow[t]{3}{*}{3} & $\bar{I}$ & 0.109 & 0.269 & $2.698(96)$ & 3 & $2.601(95)$ \\
\hline & & & II & 0.098 & 0.253 & $2.543(95)$ & 5 & $2.452(94)$ \\
\hline & & & III & 0.068 & 0.200 & $2.173(96)$ & 3 & $2.082(94)$ \\
\hline & & \multirow[t]{3}{*}{5} & I & 0.108 & 0.255 & $2.678(96)$ & 7 & $2.604(95)$ \\
\hline & & & II & 0.092 & 0.248 & $2.602(95)$ & 3 & $2.425(94)$ \\
\hline & & & III & 0.065 & 0.192 & $2.148(95)$ & 1 & $2.068(94)$ \\
\hline & \multirow[t]{6}{*}{18} & \multirow[t]{3}{*}{3} & I & 0.079 & 0.175 & $1.879(96)$ & 1 & $2.027(94)$ \\
\hline & & & II & 0.085 & 0.190 & $1.937(96)$ & 0 & $2.019(94)$ \\
\hline & & & III & 0.080 & 0.166 & $1.761(95)$ & 2 & $1.878(94)$ \\
\hline & & \multirow[t]{3}{*}{5} & I & 0.083 & 0.189 & $1.925(95)$ & 1 & $2.051(94)$ \\
\hline & & & II & 0.072 & 0.176 & $1.862(95)$ & 0 & $1.968(94)$ \\
\hline & & & III & 0.066 & 0.155 & $1.726(96)$ & 0 & $1.847(94)$ \\
\hline \multirow[t]{12}{*}{30} & \multirow[t]{6}{*}{22} & \multirow[t]{3}{*}{6} & I & 0.067 & 0.145 & $1.584(95)$ & 0 & $1.681(94)$ \\
\hline & & & II & 0.063 & 0.138 & $1.543(94)$ & 0 & $1.614(94)$ \\
\hline & & & III & 0.045 & 0.112 & $1.410(94)$ & 0 & $1.408(93)$ \\
\hline & & \multirow[t]{3}{*}{8} & $\mathrm{I}$ & 0.060 & 0.139 & $1.559(95)$ & 0 & $1.666(94)$ \\
\hline & & & II & 0.063 & 0.138 & $1.543(94)$ & 0 & $1.614(94)$ \\
\hline & & & III & 0.045 & 0.112 & $1.410(95)$ & 0 & $1.408(93)$ \\
\hline & \multirow[t]{6}{*}{26} & \multirow[t]{3}{*}{6} & I & 0.053 & 0.110 & $1.371(95)$ & 0 & $1.437(94)$ \\
\hline & & & II & 0.051 & 0.104 & $1.349(95)$ & 0 & $1.400(94)$ \\
\hline & & & III & 0.042 & 0.087 & $1.259(96)$ & 0 & $1.291(95)$ \\
\hline & & \multirow[t]{3}{*}{8} & I & 0.053 & 0.110 & $1.371(95)$ & 0 & $1.437(94)$ \\
\hline & & & II & 0.051 & 0.104 & $1.349(95)$ & 0 & $1.400(94)$ \\
\hline & & & III & 0.042 & 0.087 & $1.259(96)$ & 0 & $1.291(95)$ \\
\hline
\end{tabular}

Table 3: Results of Bayesian analysis under Prior-I for $\theta_{1}$

\begin{tabular}{|c|c|c|c|c|c|c|c|}
\hline $\mathrm{n}$ & $\mathrm{m}$ & $\mathrm{k}$ & Scheme & Bias & MSE & $\begin{array}{c}\text { Symmetric } \\
\text { Credible Interval }\end{array}$ & $\begin{array}{c}\text { HPD } \\
\text { Credible Interval }\end{array}$ \\
\hline \multirow[t]{12}{*}{20} & \multirow[t]{6}{*}{14} & \multirow[t]{3}{*}{3} & I & 0.087 & 0.164 & $1.654(96)$ & $1.486(95)$ \\
\hline & & & II & 0.080 & 0.159 & $1.616(96)$ & 1.457 (94) \\
\hline & & & III & 0.059 & 0.135 & $1.496(96)$ & $1.360(94)$ \\
\hline & & \multirow[t]{3}{*}{5} & I & 0.087 & 0.158 & $1.652(97)$ & $1.485(95)$ \\
\hline & & & II & 0.074 & 0.153 & $1.606(96)$ & 1.447 (95) \\
\hline & & & III & 0.057 & 0.131 & $1.490(96)$ & $1.356(94)$ \\
\hline & \multirow[t]{6}{*}{18} & \multirow[t]{3}{*}{3} & I & 0.070 & 0.125 & $1.431(96)$ & $1.311(95)$ \\
\hline & & & II & 0.074 & 0.131 & $1.436(96)$ & $1.316(95)$ \\
\hline & & & III & 0.073 & 0.122 & $1.377(96)$ & $1.269(95)$ \\
\hline & & \multirow[t]{3}{*}{5} & I & 0.073 & 0.133 & $1.442(96)$ & $1.320(95)$ \\
\hline & & & II & 0.064 & 0.125 & $1.414(96)$ & $1.296(95)$ \\
\hline & & & III & 0.061 & 0.116 & $1.358(96)$ & $1.252(95)$ \\
\hline \multirow[t]{12}{*}{30} & \multirow[t]{6}{*}{22} & \multirow[t]{3}{*}{6} & I & 0.062 & 0.109 & $1.288(96)$ & $1.195(94)$ \\
\hline & & & II & 0.059 & 0.106 & $1.266(95)$ & $1.176(94)$ \\
\hline & & & III & 0.045 & 0.090 & $1.178(95)$ & $1.102(94)$ \\
\hline & & \multirow[t]{3}{*}{8} & I & 0.056 & 0.106 & $1.277(95)$ & $1.185(94)$ \\
\hline & & & II & 0.059 & 0.106 & $1.266(95)$ & $1.176(94)$ \\
\hline & & & III & 0.045 & 0.090 & $1.178(95)$ & $1.102(94)$ \\
\hline & \multirow[t]{6}{*}{26} & \multirow[t]{3}{*}{6} & I & 0.052 & 0.088 & $1.168(96)$ & $1.095(95)$ \\
\hline & & & II & 0.050 & 0.085 & $1.156(95)$ & $1.084(95)$ \\
\hline & & & III & 0.043 & 0.073 & $1.092(96)$ & $1.029(95)$ \\
\hline & & \multirow[t]{3}{*}{8} & I & 0.052 & 0.088 & $1.168(96)$ & $1.095(95)$ \\
\hline & & & II & 0.050 & 0.085 & $1.156(95)$ & $1.084(95)$ \\
\hline & & & III & 0.043 & 0.073 & $1.092(96)$ & 1.029 (95) \\
\hline
\end{tabular}


Table 4: Results of Bayesian analysis under Prior-II for $\theta_{1}$

\begin{tabular}{|cccccccc|}
\hline $\mathrm{n}$ & $\mathrm{m}$ & $\mathrm{k}$ & Scheme & Bias & MSE & $\begin{array}{c}\text { Symmetric } \\
\text { Credible Interval }\end{array}$ & $\begin{array}{c}\text { HPD } \\
\text { Credible Interval }\end{array}$ \\
\hline \hline 20 & 14 & 3 & I & 0.109 & 0.269 & $1.888(94)$ & $1.658(92)$ \\
& & & II & 0.098 & 0.253 & $1.832(94)$ & $1.614(92)$ \\
& & & III & 0.068 & 0.200 & $1.653(94)$ & $1.478(92)$ \\
& & 5 & I & 0.108 & 0.255 & $1.881(94)$ & $1.654(93)$ \\
& & II & 0.092 & 0.248 & $1.819(94)$ & $1.604(92)$ \\
& & III & 0.065 & 0.192 & $1.644(94)$ & $1.471(92)$ \\
& \multirow{2}{*}{18} & 3 & I & 0.079 & 0.175 & $1.565(94)$ & $1.414(93)$ \\
& & II & 0.085 & 0.190 & $1.574(94)$ & $1.422(93)$ \\
& & III & 0.080 & 0.166 & $1.491(94)$ & $1.358(93)$ \\
& 5 & I & 0.083 & 0.189 & $1.579(94)$ & $1.425(93)$ \\
& & II & 0.072 & 0.176 & $1.541(94)$ & $1.395(93)$ \\
& & III & 0.066 & 0.155 & $1.467(94)$ & $1.337(93)$ \\
30 & \multirow{2}{*}{22} & 6 & I & 0.067 & 0.145 & $1.379(94)$ & $1.268(93)$ \\
& & II & 0.063 & 0.138 & $1.352(94)$ & $1.245(93)$ \\
& & III & 0.045 & 0.112 & $1.242(93)$ & $1.155(92)$ \\
& 8 & I & 0.060 & 0.139 & $1.365(94)$ & $1.255(93)$ \\
& & II & 0.063 & 0.138 & $1.352(94)$ & $1.245(93)$ \\
& & III & 0.045 & 0.112 & $1.242(93)$ & $1.155(92)$ \\
& \multirow{2}{*}{6} & I & I & 0.053 & 0.110 & $1.232(94)$ & $1.147(94)$ \\
& & II & 0.051 & 0.104 & $1.216(94)$ & $1.134(93)$ \\
& & III & 0.042 & 0.087 & $1.139(95)$ & $1.069(94)$ \\
& 8 & I & 0.053 & 0.110 & $1.232(94)$ & $1.147(94)$ \\
& & II & 0.051 & 0.104 & $1.216(94)$ & $1.134(93)$ \\
& & III & 0.042 & 0.087 & $1.139(95)$ & $1.069(94)$ \\
\hline
\end{tabular}

Table 5: Results of frequentist analysis for $\theta_{2}$

\begin{tabular}{|c|c|c|c|c|c|c|c|c|}
\hline $\mathrm{n}$ & $\mathrm{m}$ & $\mathrm{k}$ & Scheme & Bias & MSE & $\begin{array}{l}\text { Exact } \\
\text { CI }\end{array}$ & NECI & $\begin{array}{c}\text { Bootstrap } \\
\text { CI }\end{array}$ \\
\hline \multirow[t]{12}{*}{$\overline{20}$} & \multirow[t]{6}{*}{14} & \multirow[t]{3}{*}{3} & $\mathrm{I}$ & 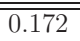 & $\overline{0.561}$ & $\overline{5.952(96)}$ & 54 & 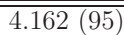 \\
\hline & & & II & 0.170 & 0.543 & $5.604(96)$ & 45 & $4.029(95)$ \\
\hline & & & III & 0.149 & 0.482 & $4.632(96)$ & 25 & $3.720(95)$ \\
\hline & & \multirow[t]{3}{*}{5} & I & 0.138 & 0.498 & $5.497(97)$ & 59 & $4.040(95)$ \\
\hline & & & II & 0.175 & 0.540 & $5.773(96)$ & 50 & $4.058(95)$ \\
\hline & & & III & 0.152 & 0.477 & $4.649(96)$ & 27 & $3.724(95)$ \\
\hline & \multirow[t]{6}{*}{18} & \multirow[t]{3}{*}{3} & I & 0.163 & 0.484 & $3.796(96)$ & 17 & $3.653(94)$ \\
\hline & & & II & 0.169 & 0.509 & $3.910(96)$ & 21 & $3.575(94)$ \\
\hline & & & III & 0.145 & 0.403 & $3.240(96)$ & 3 & $3.358(94)$ \\
\hline & & \multirow[t]{3}{*}{5} & I & 0.151 & 0.452 & $3.713(96)$ & 23 & $3.595(95)$ \\
\hline & & & II & 0.167 & 0.517 & $3.846(95)$ & 19 & $3.599(94)$ \\
\hline & & & III & 0.144 & 0.458 & $3.440(95)$ & 16 & $3.349(93)$ \\
\hline \multirow[t]{12}{*}{30} & \multirow[t]{6}{*}{22} & \multirow[t]{3}{*}{6} & I & 0.122 & 0.340 & $2.714(96)$ & 5 & $2.982(94)$ \\
\hline & & & II & 0.122 & 0.346 & $2.687(95)$ & 4 & $2.877(94)$ \\
\hline & & & III & 0.098 & 0.276 & $2.361(95)$ & 3 & $2.487(94)$ \\
\hline & & \multirow[t]{3}{*}{8} & I & 0.119 & 0.346 & $2.744(96)$ & 3 & $2.986(95)$ \\
\hline & & & II & 0.122 & 0.346 & $2.687(95)$ & 0 & $2.877(94)$ \\
\hline & & & III & 0.098 & 0.276 & $2.361(95)$ & 3 & $2.487(94)$ \\
\hline & \multirow[t]{6}{*}{26} & \multirow[t]{3}{*}{6} & I & 0.108 & 0.296 & $2.311(95)$ & 0 & $2.551(94)$ \\
\hline & & & II & 0.109 & 0.294 & $2.278(95)$ & 3 & $2.495(94)$ \\
\hline & & & III & 0.093 & 0.255 & $2.074(95)$ & 0 & $2.272(93)$ \\
\hline & & \multirow[t]{3}{*}{8} & I & 0.108 & 0.296 & $2.311(95)$ & 0 & $2.551(94)$ \\
\hline & & & II & 0.109 & 0.294 & $2.278(95)$ & 1 & $2.495(94)$ \\
\hline & & & III & 0.093 & 0.255 & $2.074(95)$ & 0 & $2.272(93)$ \\
\hline
\end{tabular}

\subsection{DATA ANALYSIS}

In this section we consider a real data set mainly for illustrative purposes. The data is taken from an experiment conducted by Dr. H.E. Walburg, Jr., of the Oak Ridge National 
Table 6: Results of Bayesian analysis under Prior-I for $\theta_{2}$

\begin{tabular}{|c|c|c|c|c|c|c|c|}
\hline $\mathrm{n}$ & $\mathrm{m}$ & $\mathrm{k}$ & Scheme & Bias & MSE & $\begin{array}{c}\text { Symmetric } \\
\text { Credible Interval }\end{array}$ & $\begin{array}{c}\text { HPD } \\
\text { Credible Interval }\end{array}$ \\
\hline \multirow[t]{12}{*}{20} & \multirow[t]{6}{*}{14} & \multirow[t]{3}{*}{3} & $\overline{I I}$ & 0.129 & 0.338 & $2.536(97)$ & $2.216(94)$ \\
\hline & & & II & 0.130 & 0.329 & $2.500(97)$ & $2.189(94)$ \\
\hline & & & III & 0.118 & 0.310 & $2.362(96)$ & $2.088(94)$ \\
\hline & & \multirow[t]{3}{*}{5} & I & 0.104 & 0.304 & $2.466(97)$ & $2.158(94)$ \\
\hline & & & II & 0.134 & 0.326 & $2.511(97)$ & $2.480(92)$ \\
\hline & & & III & 0.121 & 0.307 & $2.366(96)$ & $2.092(94)$ \\
\hline & \multirow[t]{6}{*}{18} & \multirow[t]{3}{*}{3} & I & 0.132 & 0.316 & $2.264(96)$ & $2.020(94)$ \\
\hline & & & II & 0.137 & 0.326 & $2.258(96)$ & $2.015(95)$ \\
\hline & & & III & 0.123 & 0.280 & $2.140(96)$ & $1.925(94)$ \\
\hline & & \multirow[t]{3}{*}{5} & I & 0.123 & 0.299 & $2.237(96)$ & $1.997(94)$ \\
\hline & & & II & 0.135 & 0.334 & $2.261(96)$ & $2.017(94)$ \\
\hline & & & III & 0.119 & 0.307 & $2.143(95)$ & $1.926(94)$ \\
\hline \multirow[t]{12}{*}{30} & \multirow[t]{6}{*}{22} & \multirow[t]{3}{*}{6} & I & 0.106 & 0.246 & $1.990(96)$ & $1.807(94)$ \\
\hline & & & II & 0.106 & 0.249 & $1.967(96)$ & $1.789(94)$ \\
\hline & & & III & 0.090 & 0.213 & $1.831(95)$ & $1.680(94)$ \\
\hline & & \multirow[t]{3}{*}{8} & I & 0.103 & 0.247 & $1.989(96)$ & $1.805(95)$ \\
\hline & & & II & 0.106 & 0.249 & $1.967(96)$ & $1.789(94)$ \\
\hline & & & III & 0.090 & 0.213 & $1.831(95)$ & $1.680(94)$ \\
\hline & \multirow[t]{6}{*}{26} & \multirow[t]{3}{*}{6} & I & 0.097 & 0.222 & $1.818(96)$ & $1.671(95)$ \\
\hline & & & II & 0.099 & 0.223 & $1.805(96)$ & $1.660(94)$ \\
\hline & & & III & 0.087 & 0.202 & $1.701(95)$ & $1.575(94)$ \\
\hline & & \multirow[t]{3}{*}{8} & I & 0.097 & 0.222 & $1.818(96)$ & $1.671(95)$ \\
\hline & & & II & 0.099 & 0.223 & $1.805(96)$ & $1.660(94)$ \\
\hline & & & III & 0.087 & 0.202 & $1.701(95)$ & $1.575(94)$ \\
\hline
\end{tabular}

Table 7: Results of Bayesian analysis under Prior-II for $\theta_{2}$

\begin{tabular}{|c|c|c|c|c|c|c|c|}
\hline $\mathrm{n}$ & $\mathrm{m}$ & $\mathrm{k}$ & Scheme & Bias & MSE & $\begin{array}{c}\text { Symmetric } \\
\text { Credible Interval }\end{array}$ & $\begin{array}{c}\text { HPD } \\
\text { Credible Interval }\end{array}$ \\
\hline \multirow[t]{12}{*}{20} & \multirow[t]{6}{*}{14} & \multirow[t]{3}{*}{ 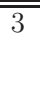 } & $\mathrm{I}$ & $\overline{0.172}$ & $\overline{0.561}$ & $2.960(95)$ & $2.509(92)$ \\
\hline & & & II & 0.170 & 0.543 & $2.902(95)$ & $2.470(92)$ \\
\hline & & & III & 0.149 & 0.482 & $2.686(94)$ & $2.318(92)$ \\
\hline & & \multirow[t]{3}{*}{5} & I & 0.138 & 0.498 & $2.852(95)$ & $2.424(91)$ \\
\hline & & & II & 0.175 & 0.540 & $2.915(95)$ & $2.480(92)$ \\
\hline & & & III & 0.152 & 0.477 & 2.691 (94) & $2.323(92)$ \\
\hline & \multirow[t]{6}{*}{18} & \multirow[t]{3}{*}{3} & I & 0.163 & 0.484 & $2.549(94)$ & $2.226(93)$ \\
\hline & & & II & 0.169 & 0.509 & $2.546(94)$ & $2.226(93)$ \\
\hline & & & III & 0.145 & 0.403 & $2.367(94)$ & $2.093(93)$ \\
\hline & & \multirow[t]{3}{*}{5} & I & 0.151 & 0.452 & $2.508(94)$ & $2.194(92)$ \\
\hline & & & II & 0.167 & 0.517 & $2.554(94)$ & $2.230(92)$ \\
\hline & & & III & 0.144 & 0.458 & $2.387(94)$ & $2.105(92)$ \\
\hline \multirow[t]{12}{*}{30} & \multirow[t]{6}{*}{22} & \multirow[t]{3}{*}{6} & I & 0.122 & 0.340 & $2.167(95)$ & $1.941(93)$ \\
\hline & & & II & 0.122 & 0.346 & $2.141(94)$ & 1.920 (93) \\
\hline & & & III & 0.098 & 0.276 & $1.960(94)$ & $1.780(92)$ \\
\hline & & \multirow[t]{3}{*}{8} & I & 0.119 & 0.346 & $2.170(95)$ & $1.942(93)$ \\
\hline & & & II & 0.122 & 0.346 & $2.141(94)$ & $1.920(93)$ \\
\hline & & & III & 0.098 & 0.276 & $1.960(94)$ & $1.780(92)$ \\
\hline & \multirow[t]{6}{*}{26} & \multirow[t]{3}{*}{6} & I & 0.108 & 0.296 & $1.949(95)$ & $1.773(94)$ \\
\hline & & & II & 0.109 & 0.294 & $1.932(94)$ & $1.760(93)$ \\
\hline & & & III & 0.093 & 0.255 & $1.801(94)$ & $1.655(93)$ \\
\hline & & \multirow[t]{3}{*}{8} & I & 0.108 & 0.296 & $1.949(95)$ & $1.773(94)$ \\
\hline & & & II & 0.109 & 0.294 & $1.932(94)$ & $1.760(93)$ \\
\hline & & & III & 0.093 & 0.255 & $1.801(94)$ & $1.655(93)$ \\
\hline
\end{tabular}


Laboratory (see Hoel [22]). A group of male mice received a radiation dose of 300r at age 5-6 weeks. The causes of deaths were classified as (1) Thymic Lymphoa, (2) Reticulum Cell Sarcoma and (3) Other causes. For our analysis we have consider Reticulum Cell Sarcoma as Cause-1 and combined all other causes as Cause-2. We have generated a generalized progressively censored sample based on the following scheme: $n=77, k=20, m=25, T=$ $700, R_{1}=\ldots=R_{24}=2, R_{25}=4$. The data set is presented below. Here the first component represents the failure time and the second component represents the cause of failure.

$(40,2),(42,2),(62,2),(163,2),(179,2),(206,2),(222,2),(228,2),(252,2),(259,2)$, $(318,1),(385,2),(407,2),(420,2),(462,2),(507,2),(517,2),(524,2),(525,1),(528,1)$, $(536,1),(605,1),(612,1),(620,2),(621,1)$.

From the data set we obtain $W=\sum_{i=1}^{m}\left(R_{i}+1\right) z_{i}=28962 . D_{1}=7, D_{2}=18$. Since we do not have any prior information about the unknown parameters, we use Prior-II in this case. The Bayes estimates of $\theta_{1}$ and $\theta_{2}$ are $\widehat{\theta}_{1 B}=4137.429$ and $\widehat{\theta}_{2 B}=1609$, respectively. These are also the MLEs of $\theta_{1}$ and $\theta_{2}$. The Bayes estimates of $\lambda_{1 B}$ and $\lambda_{2 B}$ are $\widehat{\lambda}_{1}=0.0003$ and $\widehat{\lambda}_{2}=0.0006$, respectively. We present different $95 \%$ confidence and credible intervals of $\theta_{1}$ and $\theta_{2}$ in Table 8 . In Figure 7 we present the $95 \%$ credible set of $\theta_{1}$ and $\theta_{2}$.

Table 8: Results for real data

\begin{tabular}{|c|c|c|c|}
\hline & Exact CI & Bootstrap CI & HPD CRI \\
\hline$\theta_{1}$ & $2017.686,10397.358$ & $2061.129,12220.355$ & $1715.194,7480.241$ \\
$\theta_{2}$ & $1018.497,2790.006$ & $976.663,2749.781$ & $939.656,2363.621$ \\
\hline
\end{tabular}

\section{Conclusion}

In this paper we consider the analysis of generalized progressive hybrid censored data in presence of competing risks. It is assumed that the competing causes of failures satisfy the latent failure time model assumption of Cox [15]. We further assume that the lifetime distributions 


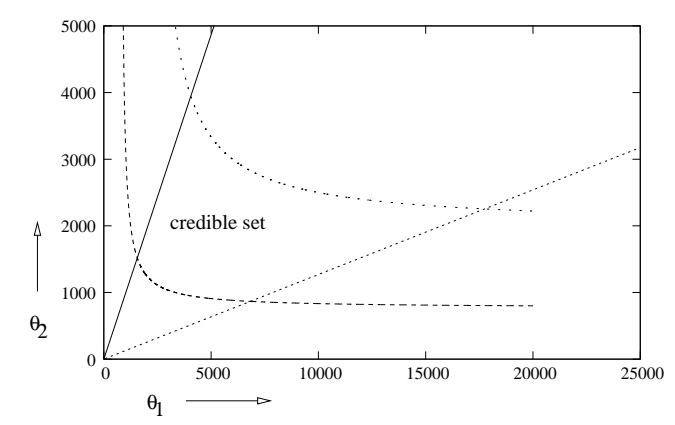

Figure 7: Credible set of $\theta_{1}$ and $\theta_{2}$

of the competing causes are exponentially distributed with different scale parameters. We carry out both the frequentist and Bayesian analysis of the unknown parameters, and it is observed that the performances of the Bayes estimates are better than the MLEs. It should be mention that recently Gorny and Cramer [20] developed the exact likelihood inference for exponential distributions under generalized hybrid censoring schemes using splines. The treatments are completely different than ours. It will be interesting to develop the exact inference results in presence of competing using splines approach. More work is needed in that direction. One natural extension of the present work is when the lifetime distributions of the competing causes follow Weibull distributions. It is a more challenging problem. Work is in progress and it will reported later.

\section{ACKNOWLEDGEMENTS:}

The would like to thank the referees for their constructive suggestions which have helped us to improve the manuscript significantly. 


\section{Appendix: The Proof of the Main Theorem}

First we derive the distribution function of $\widehat{\theta}_{1}$ which is given below.

$$
\begin{aligned}
F_{\widehat{\theta}_{1} \mid D_{1}>0}(x)= & P\left(\widehat{\theta}_{1} \leq x \mid D_{1}>0\right) \\
= & P\left(\widehat{\theta}_{1} \leq x, A \mid D_{1}>0\right)+P\left(\widehat{\theta}_{1} \leq x, B \mid D_{1}>0\right)+P\left(\widehat{\theta}_{1} \leq x, C \mid D_{1}>0\right) \\
= & \sum_{j=k}^{m-1} \sum_{i=1}^{j} P\left(\widehat{\theta}_{1} \leq x \mid J=j, D_{1}=i\right) P\left(J=j, D_{1}=i \mid D_{1}>0\right) \\
& +\sum_{i=1}^{k} P\left(\widehat{\theta}_{1} \leq x \mid B, D_{1}=i\right) P\left(B, D_{1}=i \mid D_{1}>0\right) \\
& +\sum_{i=1}^{m} P\left(\widehat{\theta}_{1} \leq x \mid C, D_{1}=i\right) P\left(C, D_{1}=i \mid D_{1}>0\right),
\end{aligned}
$$

where,

$$
A=\left\{Z_{k: m: n}<T<Z_{m: m: n}\right\}, B=\left\{T<Z_{k: m: n}<Z_{m: m: n}\right\}, C=\left\{Z_{k: m: n}<Z_{m: m: n}<T\right\} .
$$

Now to compute the terms on the right hand side of (7), we need the following Lemmas. Lemma: 1 The joint distribution of $Z_{1: m: n}, \ldots, Z_{J: m: n}$ given $J=j, D_{1}=i$ for $i=1, \ldots, j$ and $j=k, \ldots, m-1$ at $z_{1}, \ldots, z_{j}$, is given by,

$$
\begin{aligned}
& f_{Z_{1: m: n}, \ldots, Z_{j: m: n} \mid J=j, D_{1}=i}\left(z_{1}, \ldots, z_{j}\right) \\
& =\frac{\prod_{v=1}^{j} \gamma_{v}}{P\left(J=j, D_{1}=i\right)}\left(\frac{1}{\theta_{1}}\right)^{i}\left(\frac{1}{\theta_{2}}\right)^{j-i} e^{-\frac{1}{\theta}\left(\sum_{s=1}^{j} z_{s}\left(1+R_{s}\right)+T R_{j}^{*}\right) .}
\end{aligned}
$$

Proof of Lemma 1: For $j=k, k+1, \ldots m$ and $i=1,2, \ldots, j$, consider left side of (8) ),

$$
\begin{aligned}
& P\left(z_{1}<Z_{1: m: n}<z_{1}+d z_{1}, \ldots, z_{j}<Z_{j: m: n}<z_{j}+d z_{j} \mid J=j, D_{1}=i\right) \\
& =\frac{P\left(z_{1}<Z_{1: m: n}<z_{1}+d z_{1}, \ldots, z_{j}<Z_{j: m: n}<z_{j}+d z_{j}, J=j, D_{1}=i\right)}{P\left(J=j, D_{1}=i\right)}
\end{aligned}
$$

Note that, the event $\left.\left\{z_{1}<Z_{1: m: n}<z_{1}+d z_{1}, \ldots, z_{j}<Z_{j: m: n}<z_{j}+d z_{j}, J=j, D_{1}=i\right)\right\}$ for $i=1, \ldots, j ; j=k, k+1, \ldots, m$ is nothing but the failure times of $j$ units till time point 
$T$ and out of them $i$ units have failed due to Cause-1. The probability of this event is the likelihood contribution of the data when $T^{*}=T$. Thus (9) becomes,

$$
\prod_{v=1}^{j} \gamma_{v}\left(\frac{1}{\theta_{1}}\right)^{i}\left(\frac{1}{\theta_{2}}\right)^{j-i} \frac{e^{-\frac{1}{\theta}\left[\sum_{s=1}^{j}\left(1+R_{s}\right) z_{s}+T R_{j}^{*}\right]}}{P\left(J=j, D_{1}=i\right)} d z_{1} \ldots d z_{j} .
$$

Lemma 2: The joint distribution of $Z_{1: m: n}, \ldots, Z_{k: m: n}$ given $T<Z_{k: m: n}<Z_{m: m: n}, D_{1}=i$ for $i=1, \ldots, k$ at $z_{1}, \ldots, z_{k}$, is given by

$$
\begin{aligned}
& f_{Z_{1: m: n}, \ldots, Z_{k: m: n} \mid T<Z_{k: m: n}<Z_{m: m: n}, D_{1}=i}\left(z_{1}, \ldots, z_{k}\right) \\
& =\frac{\prod_{v=1}^{k} \gamma_{v}}{P\left(T<Z_{k: m: n}<Z_{m: m: n}, D_{1}=i\right)}\left(\frac{1}{\theta_{1}}\right)^{i}\left(\frac{1}{\theta_{2}}\right)^{k-i} e^{-\frac{1}{\theta}\left(\sum_{s=1}^{k-1} z_{s}\left(1+R_{s}\right)+z_{k}\left(1+R_{k}^{*}\right)\right) .}
\end{aligned}
$$

Proof of Lemma 2: For $i=1,2, \ldots, k$, consider left side of (10),

$$
\begin{aligned}
& P\left(z_{1}<Z_{1: m: n}<z_{1}+d z_{1}, \ldots, z_{k}<Z_{k: m: n}<z_{k}+d z_{k} \mid T<Z_{k: m: n}<Z_{m: m: n}, D_{1}=i\right) \\
& =\frac{P\left(z_{1}<Z_{1: m: n}<z_{1}+d z_{1}, \ldots, z_{k}<Z_{k: m: n}<z_{k}+d z_{k}, T<Z_{k: m: n}<Z_{m: m: n}, D_{1}=i\right)}{P\left(T<Z_{k: m: n}<Z_{m: m: n}, D_{1}=i\right)}
\end{aligned}
$$

Note that, the event $\left.\left\{z_{1}<Z_{1: m: n}<z_{1}+d z_{1}, \ldots, z_{k}<Z_{k: m: n}<z_{k}+d z_{k}, T<Z_{k: m: n}, D_{1}=i\right)\right\}$ for $i=1, \ldots, k$ is nothing but the failure times of $k$ units till the experiment termination point $Z_{k: m: n}$ and out of them $i$ units have failed due to Cause-1. The probability of this event is the likelihood contribution of the data when $T^{*}=Z_{k: m: n}$. Thus (11) becomes,

$$
\prod_{v=1}^{k} \gamma_{v}\left(\frac{1}{\theta_{1}}\right)^{i}\left(\frac{1}{\theta_{2}}\right)^{k-i} \frac{e^{-\frac{1}{\theta}\left[\sum_{s=1}^{k-1}\left(1+R_{s}\right) z_{s}+z_{k}\left(1+R_{k}^{*}\right)\right]}}{P\left(T<Z_{k: m: n}<Z_{m: m: n}, D_{1}=i\right)} d z_{1} \ldots d z_{k} .
$$


Lemma 3: The joint distribution of $Z_{1: m: n}, \ldots, Z_{m: m: n}$ given $Z_{k: m: n}<Z_{m: m: n}<T, D_{1}=i$ for $i=1, \ldots, m$ at $z_{1}, \ldots, z_{m}$, is given by,

$$
\begin{aligned}
& f_{Z_{1: m: n}, \ldots, Z_{m: m: n} \mid Z_{k: m: n}<Z_{m: m: n}<T, D_{1}=i}\left(z_{1}, \ldots, z_{m}\right) \\
& =\frac{\prod_{v=1}^{m} \gamma_{v}}{P\left(Z_{k: m: n}<Z_{m: m: n}<T, D_{1}=i\right)}\left(\frac{1}{\theta_{1}}\right)^{i}\left(\frac{1}{\theta_{2}}\right)^{m-i} e^{-\frac{1}{\theta} \sum_{s=1}^{m}\left(1+R_{s}\right) z_{s}} .
\end{aligned}
$$

Proof of Lemma 3: For $i=1,2, \ldots m$, consider left side of (12),

$$
\begin{aligned}
& P\left(z_{1}<Z_{1: m: n}<z_{1}+d z_{1}, \ldots, z_{m}<Z_{m: m: n}<z_{m}+d z_{m} \mid Z_{k: m: n}<Z_{m: m: n}<T, D_{1}=i\right) \\
& =\frac{P\left(z_{1}<Z_{1: m: n}<z_{1}+d z_{1}, \ldots, z_{m}<Z_{m: m: n}<z_{m}+d z_{m}, Z_{k: m: n}<Z_{m: m: n}<T, D_{1}=i\right)}{P\left(Z_{k: m: n}<Z_{m: m: n}<T, D_{1}=i\right)}
\end{aligned}
$$

Note that, the event $\left\{z_{1}<Z_{1: m: n}<z_{1}+d z_{1}, \ldots, z_{m}<Z_{m: m: n}<z_{m}+d z_{m}, Z_{k: m: n}<Z_{m: m: n}<\right.$ $\left.\left.T, D_{1}=i\right)\right\}$ for $i=1, \ldots, m$ is nothing but the failure times of $m$ units till the experiment termination point $Z_{m: m: n}$ and out of them $i$ units have failed due to Cause-1. The probability of this event is the likelihood contribution of the data when $T^{*}=Z_{m: m: n}$. Thus 13 becomes,

$$
\prod_{v=1}^{m} \gamma_{v}\left(\frac{1}{\theta_{1}}\right)^{i}\left(\frac{1}{\theta_{2}}\right)^{m-i} \frac{e^{-\frac{1}{\theta} \sum_{s=1}^{m}\left(1+R_{s}\right) z_{s}}}{P\left(Z_{k: m: n}<Z_{m: m: n}<T, D_{1}=i\right)} d z_{1} \ldots d z_{m}
$$

Theorem 3. The conditional moment generating function of $\widehat{\theta}_{1}$ given $J=j, D_{1}=i$ for $i=1, \ldots, j$ and $j=k, \ldots, m-1$ is given by

$$
\begin{aligned}
& E\left(e^{t \widehat{\theta}_{1}} \mid J=j, D_{1}=i\right) \\
= & \prod_{v=1}^{j} \frac{\gamma_{v}}{P\left(J=j, D_{1}=i\right)}\left(\frac{1}{\theta_{1}}\right)^{i}\left(\frac{1}{\theta_{2}}\right)^{j-i}\left(\frac{1}{\theta}-\frac{t}{i}\right)^{-j} \times \\
& \sum_{v=0}^{j} \frac{(-1)^{v} e^{-T\left(\frac{1}{\theta}-\frac{t}{i}\right) \gamma_{j-v+1}}}{\left\{\prod_{h=1}^{v}\left(\gamma_{j+1-v}-\gamma_{j+1-v+h}\right)\right\}\left\{\prod_{h=1}^{j-v}\left(\gamma_{h}-\gamma_{j-v+1}\right)\right\}} .
\end{aligned}
$$


Proof.

$$
\begin{aligned}
& E\left[e^{t \widehat{\theta}_{1}} \mid J=j, D_{1}=i\right] \\
& =\frac{\prod_{v=1}^{j} \gamma_{v}}{P\left(J=j, D_{1}=i\right)}\left(\frac{1}{\theta_{1}}\right)^{i}\left(\frac{1}{\theta_{2}}\right)^{j-i} \times \\
& \int_{0}^{T} \int_{0}^{z_{j}} \ldots \int_{0}^{z_{2}} e^{-z_{1}\left(1+R_{1}\right)\left(\frac{1}{\theta}-\frac{t}{i}\right)} \ldots e^{-z_{j}\left(1+R_{j}\right)\left(\frac{1}{\theta}-\frac{t}{i}\right)} e^{-T R_{j}^{*}\left(\frac{1}{\theta}-\frac{t}{i}\right)} d z_{1} \ldots d z_{j}
\end{aligned}
$$

The above equality follows using Lemma 1 ,

$$
\begin{aligned}
& =\frac{\prod_{v=1}^{j} \gamma_{v}}{P\left(J=j, D_{1}=i\right)}\left(\frac{1}{\theta_{1}}\right)^{i}\left(\frac{1}{\theta_{2}}\right)^{j-i} e^{-T R_{j}^{*}\left(\frac{1}{\theta}-\frac{t}{i}\right)} \times \\
& \int_{0}^{T} \int_{0}^{z_{j}} \ldots \int_{0}^{z_{2}} e^{-z_{1}} e^{-z_{1}\left(1+R_{1}\right)\left(\frac{1}{\theta}-\frac{t}{i}\right)-1} \ldots e^{-z_{j}} e^{-z_{j}\left(1+R_{j}\right)\left(\frac{1}{\theta}-\frac{t}{i}\right)-1} d z_{1} \ldots d z_{j} \\
= & \frac{\prod_{v=1}^{j} \gamma_{v}}{P\left(J=j, D_{1}=i\right)}\left(\frac{1}{\theta_{1}}\right)^{i}\left(\frac{1}{\theta_{2}}\right)^{j-i}\left(\frac{1}{\theta}-\frac{t}{i}\right)^{-j} \times \\
& \sum_{v=0}^{j} \frac{(-1)^{v} e^{-T\left(\frac{1}{\theta}-\frac{t}{i}\right) \gamma_{j-v+1}}}{\left\{\prod_{h=1}^{v}\left(\gamma_{j+1-v}-\gamma_{j+1-v+h}\right)\right\}\left\{\prod_{h=1}^{j-v}\left(\gamma_{h}-\gamma_{j-v+1}\right)\right\}}
\end{aligned}
$$

The last equality follows using Lemma 1 of Balakrishnan et al. [5].

Corollary 1. The conditional distribution of $\widehat{\theta}_{1}$ given $J=j, D_{1}=i$ for $i=1, \ldots, j$ and $j=k, \ldots, m-1$ is given by,

$$
\begin{aligned}
f_{\widehat{\theta}_{1} \mid J=j, D_{1}=i}(x)= & \prod_{v=1}^{j} \frac{\gamma_{v}}{P\left(J=j, D_{1}=i\right)}\left(\frac{1}{\theta_{1}}\right)^{i}\left(\frac{1}{\theta_{2}}\right)^{j-i}\left(\frac{1}{\theta}\right)^{-j} \times \\
& \sum_{v=0}^{j} \frac{(-1)^{v} e^{-\frac{T}{\theta} \gamma_{j-v+1}}}{\left\{\prod_{h=1}^{v}\left(\gamma_{j+1-v}-\gamma_{j+1-v+h}\right)\right\}\left\{\prod_{h=1}^{j-v}\left(\gamma_{h}-\gamma_{j-v+1}\right)\right\}} \times \\
& f_{G}\left(x ; \frac{T}{i} \gamma_{j-v+1}, j, \frac{i}{\theta}\right) .
\end{aligned}
$$

Theorem 4. The conditional moment generating function of $\widehat{\theta}_{1}$ given $T<Z_{k: m: n}<Z_{m: m: n}$, $D_{1}=i$ for $i=1, \ldots, k$ is given by,

$$
\begin{aligned}
& E\left(e^{t \widehat{\theta}_{1}} \mid T<Z_{k: m: n}<Z_{m: m: n}, D_{1}=i\right) \\
& =\frac{\prod_{v=1}^{k} \gamma_{v}}{P\left(T<Z_{k: m: n}<Z_{m: m: n}, D_{1}=i\right)}\left(\frac{1}{\theta_{1}}\right)^{i}\left(\frac{1}{\theta_{2}}\right)^{k-i}\left(\frac{1}{\theta}-\frac{t}{i}\right)^{-k} \times \\
& \sum_{v=0}^{k-1} \frac{(-1)^{v} e^{-T\left(\frac{1}{\theta}-\frac{t}{i}\right) \gamma_{k-v}}}{\left\{\prod_{j=1}^{v}\left(\gamma_{k-v}-\gamma_{k-v+j}\right)\right\}\left\{\prod_{j=1}^{k-1-v}\left(\gamma_{j}-\gamma_{k-v}\right)\right\} \gamma_{k-v}} .
\end{aligned}
$$


Proof.

$$
\begin{aligned}
& E\left[e^{t \widehat{\theta}_{1}} \mid T<Z_{k: m: n}<Z_{m: m: n}, D_{1}=i\right] \\
= & \frac{\prod_{v=1}^{k} \gamma_{v}}{P\left(T<Z_{k: m: n}<Z_{m: m: n}, D_{1}=i\right)}\left(\frac{1}{\theta_{1}}\right)^{i}\left(\frac{1}{\theta_{2}}\right)^{k-i} \times \\
& \int_{T}^{\infty} \int_{0}^{z_{k}} \ldots \int_{0}^{z_{2}} e^{-z_{1}\left(1+R_{1}\right)\left(\frac{1}{\theta}-\frac{t}{i}\right)} \ldots e^{-z_{k-1}\left(1+R_{k-1}\right)\left(\frac{1}{\theta}-\frac{t}{i}\right)} e^{-z_{k}\left(1+R_{k}^{*}\right)\left(\frac{1}{\theta}-\frac{t}{i}\right)} d z_{1} \ldots d z_{k}
\end{aligned}
$$

The above equality follows using Lemma 2 ,

$$
\begin{aligned}
= & \frac{\prod_{v=1}^{k} \gamma_{v}}{P\left(T<Z_{k: m: n}<Z_{m: m: n}, D_{1}=i\right)}\left(\frac{1}{\theta_{1}}\right)^{i}\left(\frac{1}{\theta_{2}}\right)^{k-i} \times \\
& \int_{T}^{\infty}\left[\int_{0}^{z_{k}} \ldots \int_{0}^{z_{2}} e^{-z_{1}} e^{-z_{1}\left(1+R_{1}\right)\left(\frac{1}{\theta}-\frac{t}{i}\right)-1} \ldots e^{-z_{k-1}} e^{-z_{k-1}\left(1+R_{k-1}\right)\left(\frac{1}{\theta}-\frac{t}{i}\right)-1} d z_{1} \ldots d z_{k-1}\right] \\
= & \frac{e^{-z_{k}\left(1+R_{k}^{*}\right)\left(\frac{1}{\theta}-\frac{t}{i}\right)} d z_{k}}{P\left(T<Z_{k: m: n}<Z_{m: m: n}, D_{1}=i\right)}\left(\frac{1}{\theta_{1}}\right)^{i}\left(\frac{1}{\theta_{2}}\right)^{k-i} \frac{1}{\left(\frac{1}{\theta}-\frac{t}{i}\right)^{k-1}} \times \\
& \int_{T}^{\infty} \sum_{v=0}^{k-1} \frac{(-1)^{v} e^{-z_{k}\left(\frac{1}{\theta}-\frac{t}{i}\right) \gamma_{v}}}{\left\{\prod_{j=1}^{v}\left(\gamma_{k-v}-\gamma_{k-v+j}\right)\right\}\left\{\prod_{j=1}^{k-1-v}\left(\gamma_{j}-\gamma_{k-v}\right)\right\}} d z_{k}
\end{aligned}
$$

The last equality follows using Lemma 1 of Balakrishnan et al. 5]

$$
\begin{aligned}
= & \frac{\prod_{v=1}^{k} \gamma_{v}}{P\left(T<Z_{k: m: n}<Z_{m: m: n}, D_{1}=i\right)}\left(\frac{1}{\theta_{1}}\right)^{i}\left(\frac{1}{\theta_{2}}\right)^{k-i}\left(\frac{1}{\theta}-\frac{t}{i}\right)^{-k} \times \\
& \sum_{v=0}^{k-1} \frac{(-1)^{v} e^{-T\left(\frac{1}{\theta}-\frac{t}{i}\right) \gamma_{k-v}}}{\left\{\prod_{j=1}^{v}\left(\gamma_{k-v}-\gamma_{k-v+j}\right)\right\}\left\{\prod_{j=1}^{k-1-v}\left(\gamma_{j}-\gamma_{k-v}\right)\right\} \gamma_{k-v}} .
\end{aligned}
$$

Corollary 2. The conditional distribution of $\widehat{\theta}_{1}$ given $T<Z_{k: m: n}<Z_{m: m: n}, D_{1}=i$ for $i=1, \ldots, k$ is given by,

$$
\begin{aligned}
f_{\widehat{\theta}_{1} \mid T<Z_{k: m: n}<Z_{m: m: n}, D_{1}=i}(x) & =\frac{\prod_{v=1}^{k} \gamma_{v}}{P\left(T<Z_{k: m: n}<Z_{m: m: n}, D_{1}=i\right)}\left(\frac{1}{\theta_{1}}\right)^{i}\left(\frac{1}{\theta_{2}}\right)^{k-i}\left(\frac{1}{\theta}\right)^{-k} \times \\
& \sum_{v=0}^{k-1} \frac{(-1)^{v} e^{-\frac{T}{\theta} \gamma_{k-v}}}{\left\{\prod_{j=1}^{v}\left(\gamma_{k-v}-\gamma_{k-v+j}\right)\right\}\left\{\prod_{j=1}^{k-1-v}\left(\gamma_{j}-\gamma_{k-v}\right)\right\} \gamma_{k-v}} \times \\
& f_{G}\left(x ; \frac{T}{i} \gamma_{k-v}, k, \frac{i}{\theta}\right) .
\end{aligned}
$$


Theorem 5. The moment generating function of $\widehat{\theta}_{1}$ given $Z_{k: m: n}<Z_{m: m: n}<T, D_{1}=i$ for $i=1, \ldots, m$ is given by,

$$
\begin{aligned}
& E\left(e^{t \widehat{\theta}_{1}} \mid Z_{k: m: n}<Z_{m: m: n}<T, D_{1}=i\right) \\
= & \frac{\prod_{v=1}^{m} \gamma_{v}}{P\left(Z_{k: m: n}<Z_{m: m: n}<T, D_{1}=i\right)}\left(\frac{1}{\theta_{1}}\right)^{i}\left(\frac{1}{\theta_{2}}\right)^{m-i}\left(\frac{1}{\theta}-\frac{t}{i}\right)^{-m} \times \\
& \sum_{v=0}^{m} \frac{(-1)^{v} e^{-T\left(\frac{1}{\theta}-\frac{t}{i}\right)\left(\gamma_{m-v+1}-\gamma_{m+1}\right)}}{\left\{\prod_{j=1}^{v}\left(\gamma_{m-v+1}-\gamma_{m-v+j+1}\right)\right\}\left\{\prod_{j=1}^{m-v}\left(\gamma_{j}-\gamma_{m-v+1}\right)\right\}} .
\end{aligned}
$$

Proof.

$$
\begin{aligned}
& E\left[e^{t \widehat{\theta}_{1}} \mid Z_{k: m: n}<Z_{m: m: n}<T, D_{1}=i\right] \\
= & \frac{\prod_{v=1}^{m} \gamma_{v}}{P\left(Z_{k: m: n}<Z_{m: m: n}<T, D_{1}=i\right)}\left(\frac{1}{\theta_{1}}\right)^{i}\left(\frac{1}{\theta_{2}}\right)^{m-i} \times \\
& \int_{0}^{T} \int_{0}^{z_{m}} \ldots \int_{0}^{z_{2}} e^{-z_{1}\left(1+R_{1}\right)\left(\frac{1}{\theta}-\frac{t}{i}\right)} \ldots e^{-z_{m-1}\left(1+R_{m-1}\right)\left(\frac{1}{\theta}-\frac{t}{i}\right)} e^{-z_{m}\left(1+R_{m}\right)\left(\frac{1}{\theta}-\frac{t}{i}\right)} d z_{1} \ldots d z_{m}
\end{aligned}
$$

The above equality follows using Lemma 3 ,

$$
\begin{aligned}
= & \frac{\prod_{v=1}^{m} \gamma_{v}}{P\left(Z_{k: m: n}<Z_{m: m: n}<T, D_{1}=i\right)}\left(\frac{1}{\theta_{1}}\right)^{i}\left(\frac{1}{\theta_{2}}\right)^{m-i} \times \\
& \int_{0}^{T} \ldots \int_{0}^{z_{2}} e^{-z_{1}} e^{-z_{1}\left(1+R_{1}\right)\left(\frac{1}{\theta}-\frac{t}{2}\right)-1} \ldots e^{-z_{m}} e^{-z_{m}\left(1+R_{m}\right)\left(\frac{1}{\theta}-\frac{t}{2}\right)-1} d z_{1} \ldots d z_{m} \\
= & \frac{\prod_{v=1}^{m} \gamma_{v}}{P\left(Z_{k: m: n}<Z_{m: m: n}<T, D_{1}=i\right)}\left(\frac{1}{\theta_{1}}\right)^{i}\left(\frac{1}{\theta_{2}}\right)^{m-i}\left(\frac{1}{\theta}-\frac{t}{i}\right)^{-m} \times \\
& \sum_{v=0}^{m} \frac{(-1)^{v} e^{-T\left(\frac{1}{\theta}-\frac{t}{i}\right)\left(\gamma_{m-v+1}-\gamma_{m+1}\right)}}{\left\{\prod_{j=1}^{v}\left(\gamma_{m-v+1}-\gamma_{m-v+j+1}\right)\right\}\left\{\prod_{j=1}^{m-v}\left(\gamma_{j}-\gamma_{m-v+1}\right)\right\}}
\end{aligned}
$$

The last equality follows using Lemma 1 of Balakrishnan et al. [5].

Corollary 3. The conditional distribution of $\widehat{\theta}_{1}$ given $Z_{k: m: n}<Z_{m: m: n}<T, D_{1}=i$ for 
$i=1, \ldots, m$ is given by

$$
\begin{aligned}
f_{\widehat{\theta}_{1} \mid Z_{k: m: n}<Z_{m: m: n}<T, D_{1}=i}(x) & =\frac{\prod_{v=1}^{m} \gamma_{v}}{P\left(Z_{k: m: n}<Z_{m: m: n}<T, D_{1}=i\right)}\left(\frac{1}{\theta_{1}}\right)^{i}\left(\frac{1}{\theta_{2}}\right)^{m-i}\left(\frac{1}{\theta}\right)^{-m} \times \\
& \sum_{v=0}^{m} \frac{(-1)^{v} e^{-\frac{T}{\theta}\left(\gamma_{m-v+1}-\gamma_{m+1}\right)}}{\left\{\prod_{j=1}^{v}\left(\gamma_{m-v+1}-\gamma_{m-v+j+1}\right)\right\}\left\{\prod_{j=1}^{m-v}\left(\gamma_{j}-\gamma_{m-v+1}\right)\right\}} \times \\
& f_{G}\left(x ; \frac{T}{i}\left(\gamma_{m-v+1}-\gamma_{m+1}\right), m, \frac{i}{\theta}\right) .
\end{aligned}
$$

Proof of Theorem 1: Combining corollaries $1-3$, we get the first part of Theorem 1.

Derivation of $P\left(D_{1}=0\right)$.

$$
\begin{aligned}
P\left(D_{1}=0\right) & =P\left(D_{1}=0, Z_{k: m: n}<T<Z_{m: m: n}\right)+P\left(D_{1}=0, T<Z_{k: m: n}<Z_{m: m: n}\right) \\
& +P\left(D_{1}=0, Z_{k: m: n}<Z_{m: m: n}<T\right) \\
& =P\left(Z_{k: m: n}<T<Z_{m: m: n}\right) P\left(D_{1}=0 \mid Z_{k: m: n}<T<Z_{m: m: n}\right) \\
& +P\left(T<Z_{k: m: n}<Z_{m: m: n}\right) P\left(D_{1}=0 \mid T<Z_{k: m: n}<Z_{m: m: n}\right) \\
& +P\left(Z_{k: m: n}<Z_{m: m: n}<T\right) P\left(D_{1}=0 \mid Z_{k: m: n}<Z_{m: m: n}<T\right) .
\end{aligned}
$$

We find each of the above probabilities separately.

$$
\begin{aligned}
& P\left(Z_{k: m: n}<T<Z_{m: m: n}\right) \\
& =\sum_{j=k}^{m-1} P\left(Z_{j: m: n}<T<Z_{j+1: m: n}\right) \\
& =\sum_{j=k}^{m-1} \prod_{v=1}^{j+1} \gamma_{v}\left(\frac{1}{\theta}\right)^{j+1} \int_{T}^{\infty} \int_{0}^{T} \ldots \int_{0}^{z_{2}} e^{-\frac{1}{\theta} \sum_{i=1}^{j} z_{i}\left(1+R_{i}\right)} e^{-\frac{1}{\theta} z_{j+1}\left(1+R_{j+1}^{*}\right)} d z_{1} \ldots d z_{j} d z_{j+1} \\
& =\sum_{j=k}^{m-1} \prod_{v=1}^{j+1} \gamma_{v} \sum_{u=0}^{j} \frac{(-1)^{u} e^{-\frac{T}{\theta} \gamma_{j-u+1}}}{\left\{\prod_{v=1}^{u}\left(\gamma_{j-u+1}-\gamma_{j+v-u+1}\right)\right\}\left\{\prod_{v=1}^{j-u}\left(\gamma_{v}-\gamma_{j-u+1}\right)\right\}\left\{\gamma_{j-u+1}-u\right\}} . \\
& P\left(D_{1}=0 \mid J=j\right)=\left(\frac{\theta_{1}}{\theta_{1}+\theta_{2}}\right)^{j} .
\end{aligned}
$$




$$
\begin{aligned}
& P\left(T<Z_{k: m: n}<Z_{m: m: n}\right) \\
& =\prod_{v=1}^{k} \gamma_{v}\left(\frac{1}{\theta}\right)^{k} \int_{T}^{\infty} \int_{0}^{z_{k}} \ldots \int_{0}^{z_{2}} e^{-\frac{1}{\theta}\left(\sum_{i=1}^{k} z_{i}\left(1+R_{i}\right)+z_{k} \gamma_{k}\right)} d z_{1} \ldots d z_{k-1} d z_{k} \\
& =\prod_{v=1}^{k} \gamma_{v} \sum_{v=0}^{k-1} \frac{(-1)^{v} e^{-\frac{T}{\theta} \gamma_{k-v}}}{\left\{\prod_{j=1}^{v}\left(\gamma_{k-v}-\gamma_{k-v+j}\right)\right\}\left\{\prod_{j=1}^{k-1-v}\left(\gamma_{j}-\gamma_{k-v}\right)\right\} \gamma_{k-v}} . \\
& P\left(Z_{k: m: n}<Z_{m: m: n}<T\right) \\
& =\prod_{v=1}^{m} \gamma_{v}\left(\frac{1}{\theta}\right)^{m} \int_{0}^{T} \int_{0}^{z_{m}} \ldots \int_{0}^{z_{2}} e^{-\frac{1}{\theta} \sum_{i=1}^{m} z_{i}\left(1+R_{i}\right)} d z_{1} \ldots d z_{m-1} d z_{m} \\
& =\prod_{v=1}^{m} \gamma_{v} \sum_{v=0}^{m} \frac{(-1)^{v} e^{-\frac{T}{\theta}\left(\gamma_{m-v+1}-\gamma_{m+1}\right)}}{\left\{\prod_{j=1}^{v}\left(\gamma_{m-v+1}-\gamma_{m-v+j+1}\right)\right\}\left\{\prod_{j=1}^{m-v}\left(\gamma_{j}-\gamma_{m-v+1}\right)\right\}} \\
& P\left(D_{1}=0 \mid T<Z_{k: m: n}<Z_{m: m: n}\right)=\left(\frac{\theta_{1}}{\theta_{1}+\theta_{2}}\right)^{k} \\
& \left.Z_{k: m: n}<Z_{m: m: n}<T\right)=\left(\frac{\theta_{1}}{\theta_{1}+\theta_{2}}\right)^{m} .
\end{aligned}
$$

\section{References}

[1] Balakrishnan, N. (2007), Progressive censoring methodology: an appraisal, Test 16 (2007), 211-259 (with discussion).

[2] Balakrishnan, N., Aggarwala, R. (2000), Progressive Censoring: Theory, Methods, and Applications, Birkhäuser.

[3] Balakrishnan, N. and Cramer, E. (2014), The art of progressive censoring, Birkhäuser, New York.

[4] Balakrishnan, N., Cramer, E. and Iliopoulos, G. (2014), "On the method of pivoting the $\mathrm{CDF}$ for exact confidence intervals with illustration for exponential mean under life-test with time constraint", Statistics and Probability Letters, 89, 124 - 130. 
[5] Balakrishnan, N., Childs, A., Chandrasekar, B. (2002), "An efficient computational method for moments of order statistics under progressive censoring", Statistics \& Probability Letters, vol. 60, 359-365.

[6] Balakrishnan, N., Kundu, D. (2013), "Hybrid censoring models, inferential results and applications", Computational Statistics and Data Analysis, vol. 57, 166-209.

[7] Balakrishnan, N., Xie, Q., Kundu, D. (2009), "Exact inference for a simple step stress model from the exponential distribution under time constraint", Annals of the Institute of Statistical Mathematics, vol. 61, 251-274.

[8] Bartholmew, D.J. (1963), "The sampling distribution of an estimate arising in life testing", Technometrics, vol. 5, 361-372.

[9] Bhattacharya, S., Pradhan, B., Kundu,D., (2014), "Analysis of hybrid censored competing risks data", Statistics, vol. 48, No. 5, 1138-1154.

[10] Chan, P., Ng, H., Su, F. (2015), "Exact likelihood inference for the two-parameter exponential distribution under Type-II progressively hybrid censoring", Metrika, vol. 78, $747-770$.

[11] Chen, S.M., Bhattayacharya, G.K. (1998), "Exact confidence bound for an exponential parameter under hybrid censoring", Communication in Statistics Theory and Methodology, 2429-2442.

[12] Childs, A., Chandrasekhar, B., Balakrishnan, N., Kundu, D. (2003), "Exact likelihood inference based on Type-I and Type-II hybrid censored samples from the exponential distribution", Ann. Inst. Statist. Math., vol. 55, 319-330. 
[13] Cho, Y., Sun, H., Lee, K. (2015), "Exact likelihood inference for an exponential parameter under generalized progressive hybrid censoring scheme", Statistical Methodology, vol. $23,18-34$.

[14] Cohen, A.C. (1963), "Progressively censored samples in life testing", Technometrics, vol. 5, 327-329.

[15] Cox, D.R. (1959), "The analysis of exponentially lifetime distributed lifetime with two types of failures", Journal of Royal Statistical Society, Ser. B, vol. 21, 411-421.

[16] Cramer, E., Balakrishnan, N. (2013), "On some exact distributional results based on Type-I progressively hybrid censored data from exponential distribution", Statistical Methodology, vol. 10, 128-150.

[17] Crowder, M. (2001), Classical Competing Risks, Chapman \& Hall/CRC.

[18] Dube, S., Pradhan, B., Kundu, D. (2011), "Parameter estimation of the hybrid censored log normal distribution", Journal of Statistical Computation and Simulation, vol. 81, 275287.

[19] Epstein, B. (1954), Truncated life tests in the exponential case, Ann. Math. Statist., vol. $25,555-564$.

[20] Gorny, J. and Cramer, E. (2016), "Exact likelihood inference for exponential distribution under generalized progressive hybrid censoring schemes", Statisical Methodology, 29, 70 $-94$.

[21] Hemmati, F., Khorram, E. (2013), "Statistical analysis of log-normal distribution under type-II progressive hybrid censoring schemes", Communications in Statistics-Simulation and Computation, vol. 42, 52-75. 
[22] Hoel, D.G. (1972), "A representation of mortality data by competing risks" Biometrics, vol. $28,475-488$.

[23] Kalbfleish, J.D., Prentice, R.L. (1980), "The Statistical Analysis of The Failure Time Data”, Wiley, New York.

[24] Kundu, D. (2007), "On hybrid censored Weibull distribution", Journal of Statistical Planning and Inference, vol. 87, 221-239.

[25] Kundu, D., Basu, S. (2000), "Analysis of incomplete data in presence of competing risks", Journal of Statistical Planning and Inference, vol. 87, 221-239.

[26] Kundu, D., Gupta, R.D. (2007), "Analysis of Hybrid Life-tests in Presence of Competing Risks", Metrika, vol. 65, Issue 2, 159-170.

[27] Kundu, D., Joarder, A. (2006), "Analysis of Type-II progressively hybrid censored data", Computational Statistics and Data Analysis, 2509-2528.

[28] Kundu, D., Pradhan, B. (2011), "Bayesian analysis of progressively censored competing risks data", Sankhya, Ser. B, vol. 73, 276-296.

[29] Pena, E.A., Gupta, A.K. (1990), "Bayes estimation for the Marshall-Olkin exponential distribution", Journal of the Royal Statistical Society, Ser. B, vol. 52, 379-389.

[30] Lawless, J.F., (1982) "Statistical Models and Methods for Lifetimes Data", Wiley, New York.

[31] Prentice, R.L., Kalbfleish, J.D., Peterson, Jr. A.V., Flurnoy, N., Farewell, V.T. and Breslow, N.E. (1978), "The analysis of failure time points in presence of competing risks", Biometrics, vol. 34, 541-554. 